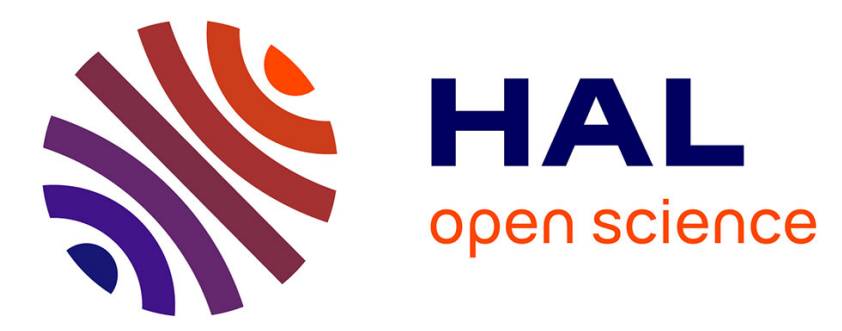

\title{
Calcul d'un spectre synthétique d'absorption à basse résolution pour un mélange CO2-CO-gaz neutre à haute température
}

J. Taine, D. Miniscloux

\section{- To cite this version:}

J. Taine, D. Miniscloux. Calcul d'un spectre synthétique d'absorption à basse résolution pour un mélange CO2-CO-gaz neutre à haute température. Revue de Physique Appliquée, 1981, 16 (11), pp.615-627. 10.1051/rphysap:019810016011061500 . jpa-00244954

\section{HAL Id: jpa-00244954 https://hal.science/jpa-00244954}

Submitted on 1 Jan 1981

HAL is a multi-disciplinary open access archive for the deposit and dissemination of scientific research documents, whether they are published or not. The documents may come from teaching and research institutions in France or abroad, or from public or private research centers.
L'archive ouverte pluridisciplinaire HAL, est destinée au dépôt et à la diffusion de documents scientifiques de niveau recherche, publiés ou non, émanant des établissements d'enseignement et de recherche français ou étrangers, des laboratoires publics ou privés. 


\title{
Calcul d'un spectre synthétique d'absorption à basse résolution pour un mélange $\mathrm{CO}_{2}$ - $\mathrm{CO}$-gaz neutre à haute température
}

\author{
J. Taine et D. Miniscloux $\left(^{*}\right)$ \\ Groupe de Recherche 14 du C.N.R.S. associé à l'Ecole Centrale des Arts et Manufactures (E.C.P.), \\ Grande Voie des Vignes, 92290 Châtenay Malabry, France
}

(Reçu le 13 avril 1981, révisé le 17 juillet 1981, accepté le 22 juillet 1981)

\begin{abstract}
Résumé. - L'absorptivité monochromatique, avec une résolution de 1 à $10 \mathrm{~cm}^{-1}$, d'un mélange de gaz purs, contenant $\mathrm{CO}_{2}$, un diatomique hétéronucléaire (CO...) et éventuellement un gaz transparent dans l'infrarouge, est calculée à partir de données spectroscopiques récentes. Les spectres d'absorption, obtenus par cette méthode, sont en bon accord avec des spectres expérimentaux disponibles dans la bibliographie et correspondant à diverses longueurs de la colonne et à des valeurs de la pression totale et de la température s'étendant respectivement de $10^{4}$ à $10^{6} \mathrm{~Pa}$ et de 800 à $1500 \mathrm{~K}$.
\end{abstract}

\begin{abstract}
The monochromatic absorptivity of a mixture of pure gases containing $\mathrm{CO}_{2}$, a diatomic heteronuclear gas (e.g. CO), and possibly a gas transparent in the infrared is computed from the latest spectroscopic data with a resolution chosen within the range of 1 to $10 \mathrm{~cm}^{-1}$. The absorption spectra obtained by this method are in good agreement with the experimental spectra available in the bibliography, related to any length of the cell as well as to different values of the total pressure from $10^{4}$ to $10^{6} \mathrm{~Pa}$ and to different temperatures ranging from 800 to $1500 \mathrm{~K}$.
\end{abstract}

\section{Nomenclature}

$B_{v}, B_{v_{\mathrm{c}}}, B_{v_{\mathrm{d}}}$

$B_{v_{i}^{\prime \prime} I_{i}^{\prime \prime} \rightarrow v_{i}^{i} J_{i}^{\prime}}$

$B_{v_{i} J_{i} \rightarrow v_{i}^{\prime \prime} J_{i}^{\prime \prime}}$

$c$

$d$

$D_{v}, D_{v_{\mathrm{c}}}, D_{v_{\mathrm{d}}}$

$E_{v_{\mathrm{i}}}, E_{v_{\mathrm{i}} J_{\mathrm{i}}}, E_{J_{\mathrm{i}}}, E_{v_{\mathrm{i}}^{\prime \prime}} \cdots$

$F_{\mathrm{i}}\left(v^{\prime}\right)$

$g_{\mathrm{i}}, g_{\mathrm{i}}^{\prime \prime}, g_{\mathrm{i}}^{\prime}$

$g_{v_{\mathrm{i}}}, g_{v_{\mathrm{i}}^{\prime \prime}}, g_{v_{\mathrm{i}}^{\prime}}$

$h$

$J_{\mathrm{i}}, J_{\mathrm{i}}^{\prime}, J_{\mathrm{i}}^{\prime \prime}, J$

$k$

$l$

$L_{\mathrm{R}}$

$L_{\gamma^{\prime}}^{(\text {(I) }}$

constante rotationnelle d'un niveau. coefficient d'Einstein d'absorption. coefficient d' Einstein d'émission induite. célérité du rayonnement dans le vide. longueur de la colonne gazeuse élémentaire. constante rotationnelle d'un niveau.

énergies de vibration, de vibration-rotation, de rotation, de vibration... d'un niveau.

profil normalisé d'une raie.

dégénérescence d'un niveau de vibration-rotation.

dégénérescence d'un niveau de vibration.

constante de Planck.

nombre quantique de rotation (ou niveau de rotation).

constante de Boltzmann.

nombre quantique du moment cinétique vibratoire.

poids statistique d'une raie.

luminance monochromatique du rayonnement incident.

${ }^{*}$ ) Une partie importante de ce travail a fait l'objet du stage de fin d'études de D. Miniscloux pour obtenir le titre d'Ingénieur E.C.P. et le D.E.A. Thermique. 
$M_{\mathrm{i}}$

$n_{\mathrm{a}}$

$N_{\mathrm{i}}$

$N_{\mathrm{i}}^{\prime \prime} \ldots$

$P, P_{\mathrm{s}}$

$Q_{\mathrm{i}}(T), Q_{\mathrm{R}_{\mathrm{i}}}(T), Q_{v_{\mathrm{i}}}(T)$

$S_{v^{\prime} \mathbf{i}}$

$S_{v_{i}^{\prime \prime} v_{i}^{\prime}}$

$S_{v_{01}}\left(T_{\mathrm{S}}\right)$

$T, T_{\mathrm{S}}$

$v_{\mathrm{i}}, v_{\mathrm{i}}^{\prime}, v_{\mathrm{i}}^{\prime \prime}$

$v_{\mathrm{i}}^{\prime \prime} \rightarrow v_{\mathrm{i}}^{\prime}$

$v_{\mathrm{i}} J_{\mathrm{i}}$

$v_{\mathrm{i}}^{\prime \prime} J_{i}^{\prime \prime} \rightarrow v_{\mathrm{i}}^{\prime} J_{\mathrm{i}}^{\prime}$

$\left\langle v_{\mathrm{i}}^{\prime} J_{\mathrm{i}}^{\prime}\left|\bar{\mu}_{\mathrm{i}}\right| v_{\mathrm{i}}^{\prime \prime} J_{\mathrm{i}}^{\prime \prime}\right\rangle$

$\left\langle v_{\mathrm{i}}^{\prime}\left|q_{v}\right| v_{\mathrm{i}}^{\prime \prime}\right\rangle,\left\langle J_{\mathrm{i}}^{\prime}\left|q_{\mathrm{i}}\right| J_{\mathrm{i}}^{\prime \prime}\right\rangle$ dv

$x_{\mathrm{i}}$

$y_{\mathrm{j}^{\prime}}$

Caractères grecs :

masse molaire du constituant $i$.

nombre d'espèces de molécules absorbantes.

nombre de molécules du constituant i par unité du volume.

nombre de molécules occupant un niveau $v_{\mathrm{i}}^{\prime \prime} J_{\mathrm{i}}^{\prime \prime}$ par unité de volume...

pression totale, pression standard.

fonctions de partition de vibration-rotation, de rotation, de vibration du cons-

tituant $\mathrm{i}$.

intensité intégrée d'une raie.

intensité intégrée d'une bande.

intensité intégrée standard (à $T_{\mathrm{S}}$ et $P_{\mathrm{S}}$ ) d'une bande du constituant $\mathrm{i}$, divisée par le nombre de molécules par unité de volume.

température, température standard (296 K).

nombre quantique de vibration (niveau de vibration).

bande de vibration.

niveau de vibration-rotation.

raie d'un constituant $i$.

élément de matrice de l'opérateur dipolaire électrique.

facteurs de l'élément précédent.

élément de volume.

fraction molaire d'un constituant absorbant i.

fraction molaire d'un constituant transparent $\mathrm{j}^{\prime}$.

absorptivité monochromatique, à haute résolution, à basse résolution, thermique. demi-largeurs Lorentz, Doppler (à mi-hauteur).

paramètres d'élargissement d'une raie du constituant i par i' ou j'.

résolution de l'intégration numérique.

coefficient monochromatique d'absorption du mélange gazeux.

coefficient monochromatique d'absorption d'une raie du constituant $i$.

coefficient de Herman-Wallis.

nombre d'onde $\left(\mathrm{en}^{-1}\right)$.

bornes d'un intervalle spectral.

nombre d'onde du centre d'une raie d'absorption du constituant $i$.

nombre d'onde du centre d'une bande d'absorption.

flux directionnel monochromatique absorbé dans dV.

angle solide élémentaire.

Divers :

exposant '

exposant"

indice 0

indice $\mathbf{B}$

indices $i, i^{\prime}$

indices $j, j^{\prime}$

indice $\mathbf{S}$

relatif au niveau supérieur.

relatif au niveau inférieur.

relatif au centre d'une raie.

relatif à une bande.

relatif à un constituant absorbant.

relatif à un constituant non absorbant.

relatif aux conditions standard $\left(T_{\mathrm{S}}=296 \mathrm{~K}, P_{\mathrm{S}}=1,02 \times 10^{5} \mathrm{~Pa}\right)$.

1. Introduction. - Les transferts d'énergie par rayonnement au sein d'un mélange gazeux ou entre gaz et parois interviennent dans de nombreuses applications industrielles : fours, combustion, problèmes de sûreté nucléaire dans le cas de certains types de réacteurs... Ils sont le plus souvent couplés à d'autres transferts qui ne seront pas envisagés dans cette étude, qu'ils soient d'origine radiative, avec des suies ou des gouttelettes par exemple, ou d'origine convective. Le présent travail a pour but de contribuer à donner aux thermiciens le moyen de calculer les transferts radiatifs dans les gaz chauds avec un degré d'approximation raisonnable (5 à $10 \%)$.

Nous nous limitons à la détermination de l'absorptivité monochromatique à basse résolution (de l'ordre du $\mathrm{cm}^{-1}$ ) notée $\alpha_{v^{\prime}}^{\text {br }}$ d'une colonne de longueur $d$ (ou d'un hémisphère équivalent de rayon $d$ [1]), constituée par un mélange de gaz de température $T$, pression totale $P$ et fractions molaires $x_{i}$ connues. En effet l'absorptivité $\alpha_{v^{\prime}}^{\text {br }}$ est la donnée fondamentale, 
qui fait le plus souvent défaut dans les types d'applications précédemment cités. A partir d'elle, il est possible d'évaluer les énergies transférées entre des cellules élémentaires de gaz, isothermes ou à des températures différentes, ou entre cellules de gaz et éléments de surface isothermes de paroi; le principe d'un tel calcul est exposé dans la référence [1] par exemple. La connaissance de $\alpha_{v^{\prime}}^{\text {br }}$ permet d'abandonner l'hypothèse effarante d'un gaz gris (c'est-à-dire d'absorptivité indépendante de la longueur d'onde), hypothèse complètement erronée mais néanmoins très souvent utilisée à partir d'abaques d'émissivité hémisphérique totale.

Un certain nombre de travaux ont été publiés, il y a une vingtaine d'années, par Edwards $[2,3]$ et Ben Arieh [4], d'autres récemment par Bernstein et al. $[5,6]$, conduisant à la détermination de $\alpha_{v^{\prime}}^{\text {br }}$. Il s'agit dans les deux cas de modèles de bandes, complexes, alliant des notions de base de spectroscopie à des considérations plus ou moins empiriques tirées de l'expérience; des approximations sont introduites dans la modélisation elle-même. Le modèle d'Edwards, assez couramment utilisé par les thermiciens, se révèle satisfaisant pour obtenir la surface $A$ des bandes d'absorption, appelée bande noire équivalente ou largeur équivalente, soit :

$$
A=\int_{v_{1}^{\prime}}^{v_{2}^{\prime}} \alpha_{v^{\prime}}^{\text {br }} \mathrm{d} v^{\prime}
$$

dans laquelle $v_{1}^{\prime}$ et $v_{2}^{\prime}$ représentent les nombres d'onde bornant la bande d'absorption. Il est, par contre, assez approximatif dans la détermination de la position et du profil des bandes. Ce modèle présentait, il y a une vingtaine d'années, l'intérêt d'approcher les résultats par des expressions analytiques simples.

La méthode proposée ici est de nature différente; elle est fondée sur les récents progrès réalisés dans la détermination des intensités intégrées des bandes d'absorption des constituants de l'atmosphère $[7,8]$. A partir de ces données sûres et en utilisant des hypothèses réalistes, en particulier sur les évolutions des profils de raie en fonction de la température, nous obtenons un spectre à basse résolution, après avoir reconstitué un spectre raie par raie dans des intervalles discrets de nombres d'onde; le temps de calcul est minimisé en éliminant les contributions des raies peu intenses en un point du spectre ( $\$ 2$ et 3 ). L'intérêt de la méthode suivie est d'être évolutive puisqu'elle permet de profiter des progrès à venir dans la connaissance des données spectroscopiques à température et pression élevées; l'influence des données utilisées est examinée dans le paragraphe 4.

Nous avons confronté avec succès les résultats de cette méthode avec les spectres expérimentaux dont nous disposons ( $(5)$; ceux-ci sont relativement rares pour des températures et pressions élevées (jusqu'à $1500 \mathrm{~K}$ et $10^{6} \mathrm{~Pa}$ ). Ces spectres ont été obtenus dans des conditions de laboratoire avec une précision rela- tive de quelques pour cent et une température définie à $10 \mathrm{~K}$ près environ. Nous discuterons aussi le cas de spectres moins sûrs, obtenus à température beaucoup plus élevée, à travers la flamme de combustion du kérosène. Les températures et pression de $1500 \mathrm{~K}$ et $10^{6} \mathrm{~Pa}$, mentionnées ci-dessus, ne constituent pas a priori des limitations supérieures de la méthode utilisée; celle-ci présente de plus l'avantage sur les autres modèles cités de demeurer valable pour des température et pression beaucoup plus faibles, même dans les domaines correspondant au profil Doppler. Un autre avantage de la méthode est de s'appliquer à des colonnes gazeuses de dimensions très variées : d'une fraction de mm à plusieurs mètres. En effet, les contributions des ailes de raies dont les centres sont très éloignés du nombre d'onde considéré sont prises en compte dans le calcul, si l'épaisseur optique du milieu est importante.

Le point critique de la méthode est cependant l'absence de données sûres, concernant le paramètre d'élargissement des raies par collisions, à température élevée : nous nous sommes bornés à extrapoler, à toutes températures, une loi d'évolution [7] valable en dessous de $350 \mathrm{~K}$. Le résultat obtenu est néanmoins satisfaisant; une discussion détaillée de ce point est donnée dans le paragraphe 5 .

2. Position du problème et principe des approximations. - L'absorption d'un rayonnement infrarouge, de nombre d'onde $v^{\prime}$ donné, par les molécules du constituant i d'un mélange gazeux, provoque le passage de ces molécules d'un niveau quantifié de vibration-rotation $v_{\mathrm{i}}^{\prime \prime} J_{\mathrm{i}}^{\prime \prime}$ à un niveau $v_{\mathrm{i}}^{\prime} J_{\mathrm{i}}^{\prime}$. Le profil d'absorption, associé à la transition $v_{\mathrm{i}}^{\prime \prime} J_{\mathrm{i}}^{\prime \prime} \rightarrow v_{\mathrm{i}}^{\prime} \mathrm{J}_{\mathrm{i}}^{\prime}$, est appelé raie d'absorption; cette dernière est centrée sur le nombre d'onde $v_{0}^{\prime v_{1}^{\prime} J_{i}^{\prime \prime} v_{i}^{\prime} J_{i}^{\prime}}$, noté plus simplement $v_{0 i}^{\prime}$ dans la suite, qui correspond exactement à l'écart énergétique entre les niveaux $v_{\mathrm{i}}^{\prime \prime} J_{\mathrm{i}}^{\prime \prime}$ et $v_{\mathrm{i}}^{\prime} J_{\mathrm{i}}^{\prime}$. Le profil de la raie en fonction de $v^{\prime}$ est caractérisé par une fonction normalisée $F_{\mathrm{i}}\left(v^{\prime}\right)$ qui dépend de la température $T$, de la pression totale $P$, des fractions molaires des constituants absorbants $x_{\mathrm{i}}$ et non absorbants $y_{\mathrm{j}}$; les différents profils rencontrés (Lorentz, Doppler, Voigt...) seront abordés dans le paragraphe 4. L'ensemble des raies, correspondant à une transition entre niveaux de vibration $v_{\mathrm{i}}^{\prime \prime}$ et $v_{\mathrm{i}}^{\prime}$, constitue une bande, formée de différentes branches qui dépendent des règles de sélection sur les nombres quantiques $J_{i}^{\prime \prime}$ et $J_{\mathrm{i}}^{\prime}$ :

$$
\begin{array}{ll}
\text { - branche } \mathrm{R}: & J_{i}^{\prime}-J_{i}^{\prime \prime}=1, \\
\text { - branche } \mathrm{Q}: & J_{i}^{\prime}-J_{i}^{\prime \prime}=0, \\
\text { - branche } \mathrm{P}: & J_{i}^{\prime}-J_{i}^{\prime \prime}=-1 .
\end{array}
$$

Le spectre d'absorption d'un mélange gazeux est formé de l'ensemble des raies appartenant à toutes les bandes des différents constituants absorbants $i$; celles-ci s'interpénètrent dans un intervalle spectral $\left[v_{1}^{\prime}, v_{2}^{\prime}\right]$ (Fig. 1).

Le but de cette étude est de calculer l'absorptivité 


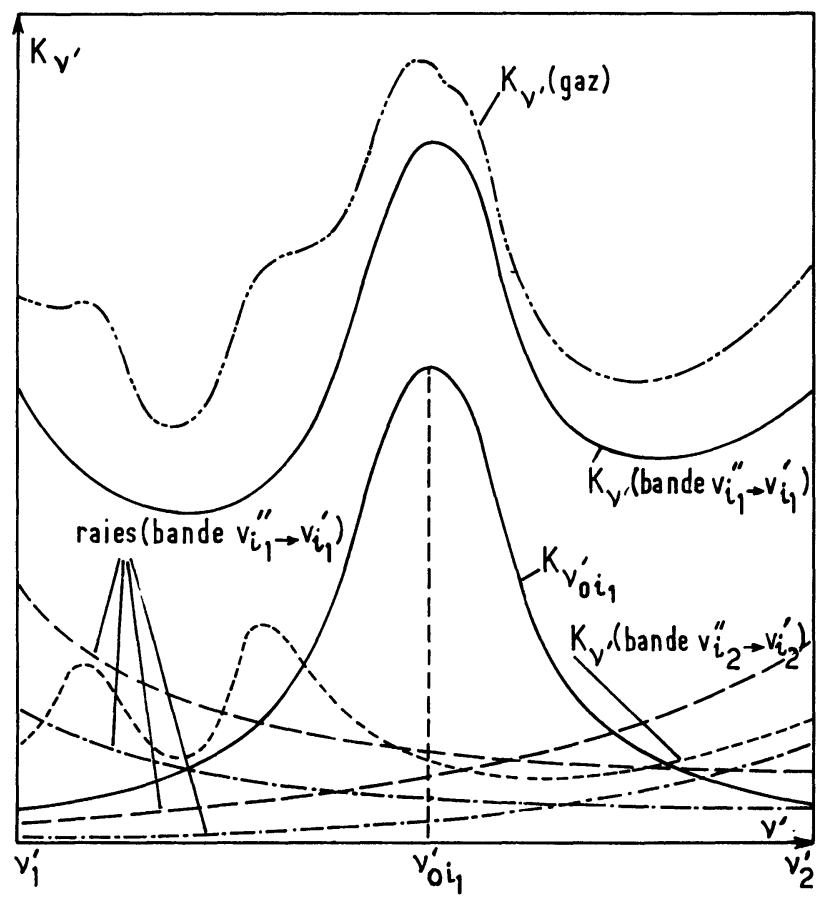

Fig. 1. - Coefficients monochromatiques d'absorption : de différentes raies de la bande $v_{\mathbf{i} 1}^{\prime \prime} \rightarrow v_{\mathbf{i} 1}^{\prime}$; de différentes bandes $v_{\mathbf{i} 1}^{\prime \prime} \rightarrow v_{\mathbf{i} 1}^{\prime}$, $v_{\mathrm{i} 2}^{\prime \prime} \rightarrow v_{\mathrm{i} 2}^{\prime} ;$ du mélange gazeux $\kappa_{v^{\prime}}$ (gaz).

[Spectral absorption coefficients : for different lines of the $v_{i 1}^{\prime}$ band ; for different bands $v_{\mathbf{i} 1}^{\prime \prime} \rightarrow v_{\mathbf{i} 1}^{\prime}$ and $v_{\mathbf{i} 2}^{\prime \prime} \rightarrow v_{\mathbf{i} 2}^{\prime}$; for the gas mixture $\kappa_{v^{\prime}}($ gas).]

monochromatique $\alpha_{v^{\prime}}$ d'une colonne gazeuse élémentaire de longueur $d$, à la température $T$ et à la pression totale $P$, connaissant les différentes fractions molaires $x_{\mathrm{i}}$ et $y_{\mathbf{j}}$ en constituants absorbants et non absorbants $[1] ; \alpha_{v^{\prime}}$ s'écrit :

$$
\begin{aligned}
& \alpha_{v^{\prime}}\left(v^{\prime}, T, x_{\mathrm{i}} \ldots y_{\mathrm{j}} \ldots P, d\right)= \\
& \quad=1-\exp \left[-d \kappa_{v^{\prime}}\left(v^{\prime}, T, x_{\mathrm{i}} \ldots y_{\mathrm{j}} \ldots, P\right)\right]
\end{aligned}
$$

où $\kappa_{v^{\prime}}$ représente le coefficient monochromatique d'absorption du mélange gazeux, donné par :

$$
\kappa_{v^{\prime}}=\sum_{\text {constituants }} \sum_{\substack{\mathrm{i} \\ v_{\mathrm{i}}^{\prime \prime} \rightarrow v_{\mathrm{i}}^{\prime}}} \sum_{\substack{\text { raies } \\ J_{i}^{\prime \prime} \rightarrow J_{\mathrm{i}}^{\prime}}} \kappa_{v_{01}^{\prime}}\left(v^{\prime}\right)
$$

si $\kappa_{v_{0 i}^{\prime}}\left(v^{\prime}\right)$ désigne, en notation simplifiée, le coefficient d'absorption de la raie centrée en $v_{0 \mathrm{i}}^{\prime}$.

La première partie du calcul consiste donc à générer, pour une bande donnée $v_{\mathrm{i}}^{\prime \prime} \rightarrow v_{\mathrm{i}}^{\prime}$, toutes les raies actives $v_{\mathrm{i}}^{\prime \prime} J_{\mathrm{i}}^{\prime \prime} \rightarrow v_{\mathrm{i}}^{\prime} J_{\mathrm{i}}^{\prime}$, puis à déterminer pour chacune d'elles $v_{0 \mathrm{i}}^{\prime}$ et $\kappa_{v_{01}^{\prime}}$. Parmi toutes les bandes d'absorption de la bibliographie $[7,8]$, nous n'avons retenu que celles dont les intensités intégrées $S_{v_{i}^{\prime \prime} v_{i}^{\prime}}$ vérifient la relation :

$$
S_{v_{i}^{\prime \prime} v_{\mathrm{i}}}\left(T, P, d, x_{\mathrm{i}} \ldots\right)>C .
$$

La quantité $C$ dépend des conditions et est choisie de façon qu'une bande ayant l'intensité intégrée $C$ ne contribue à l'absorptivité que pour moins de $10^{-3}$.
L'expression de $S_{v_{i}^{\prime \prime} v_{\mathrm{i}}^{\prime}}$ est donnée dans le paragraphe 4, en fonction des données expérimentales. Une deuxième approximation.consiste lors de la génération des raies à éliminer toutes celles, peu intenses, telles que :

$$
\mathrm{d} \kappa_{v_{01}}\left(v_{0 \mathrm{i}}^{\prime}\right)<\eta_{1} .
$$

Le paramètre $\eta_{1}$ est pris, en général, entre $10^{-5}$ et $10^{-7}$ suivant les conditions considérées et le nombre de raies éliminées de ce fait. L'erreur absolue sur $\alpha_{v^{\prime}}^{\text {br }}$, due à cette approximation, est inférieure à $10^{-3}$.

Le but de ce calcul n'est pas d'obtenir l'intégralité d'un spectre à haute résolution $\alpha_{v^{\prime}}^{\text {hr }}$ (Fig. 2a), à partir de (2) et (3), pour tout nombre d'onde $v^{\prime}$, mais d'obtenir un spectre à basse résolution, de l'ordre $\mathrm{du} \mathrm{cm}^{-1}$,

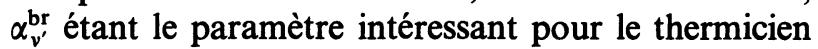
(Fig. 2b). Nous utilisons donc les relations (2) et (3) qu'en des intervalles spectraux, de largeurs $\Delta v^{\prime}$ (de l'ordre $\mathrm{du} \mathrm{cm}^{-1}$ ), centrés en des points $v_{\mathbf{k}}^{\prime}$ de l'intervalle $\left[v_{1}^{\prime}, v_{2}^{\prime}\right]$, équidistants de $20 \mathrm{~cm}^{-1}$ dans ce calcul.

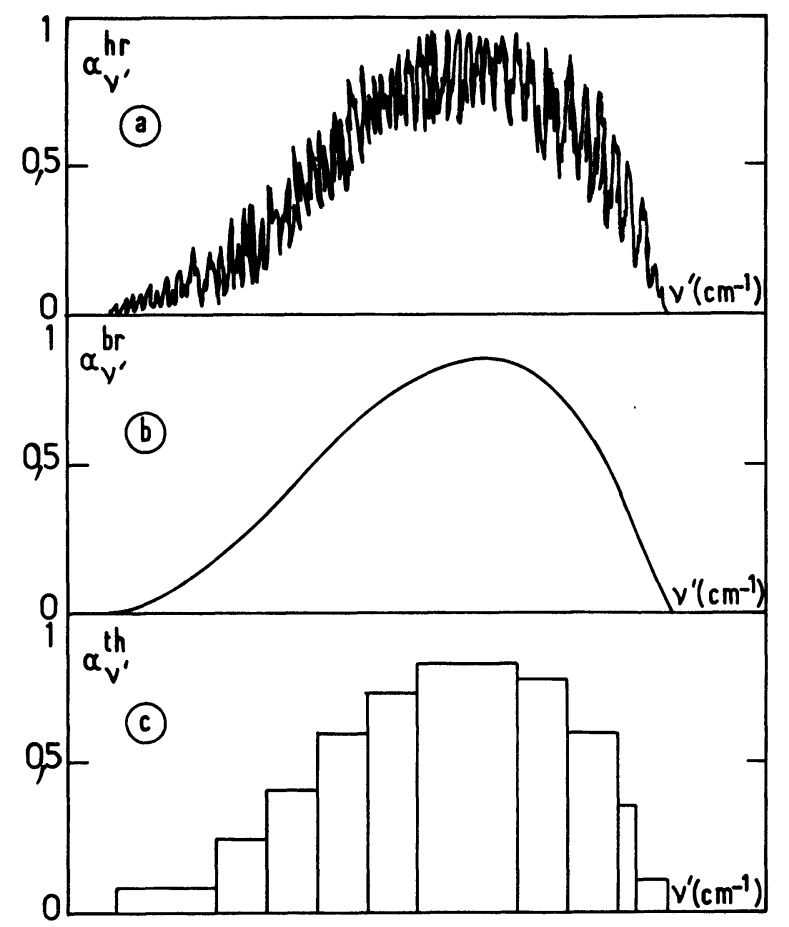

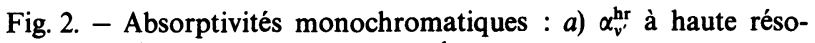

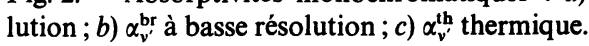

[Spectral absorptivities : a) $\alpha_{v^{\prime}}^{\text {hr }}$ at high resolution; $b$ ) $\alpha_{v}^{\text {br }}$ at low resolution; $c) \alpha_{v^{\prime}}^{\text {th }}$ thermal.]

Nous appliquons la relation :

$$
\alpha_{v^{\prime}}^{\mathrm{br}}=\left(\Delta v^{\prime}\right)^{-1} \int_{v_{\mathbf{k}^{\prime}}-\frac{\Delta v^{\prime}}{2}}^{v_{\mathbf{k}}+\frac{\Delta v^{\prime}}{2}}\left(1-\exp \left[-\mathrm{d} \kappa_{v^{\prime}}\right]\right) \mathrm{d} v^{\prime} .
$$

L'intervalle $\Delta v^{\prime}$ a été choisi suffisamment petit, pour que, dans les calculs de transferts radiatifs, on puisse considérer que la luminance monochromatique du rayonnement noir reste constante sur $\Delta v^{\prime}$ et suffisamment grand pour éliminer la structure fine du 
spectre. Deux nouvelles approximations sont réalisées à partir de (6) :

- L'intégrale est calculée numériquement avec un pas inférieur au dixième de la largeur d'une raie.

- On ne prend en compte dans l'expression de $\mathrm{d} \kappa_{v^{\prime}}$, en un point $v^{\prime}$ de l'intervalle

$$
\left[v_{\mathbf{k}}^{\prime}-\frac{\Delta v^{\prime}}{2}, v_{\mathbf{k}}^{\prime}+\frac{\Delta v^{\prime}}{2}\right],
$$

que les raies vérifiant :

$$
\mathrm{d} \kappa_{v_{01}^{\prime}}\left(v_{\mathbf{k}}^{\prime}\right)<\eta_{2} .
$$

Le paramètre $\eta_{2}$ est compris entre $10^{-6}$ et $10^{-8}$ suivant les conditions. L'incertitude absolue sur l'absorptivité, due à cette approximation, est inférieure à $10^{-3}$.

3. Génération des raies. - Le nombre d'onde $v_{0}^{\prime}$ du centre d'une raie, donné par :

$$
h c v_{0 \mathrm{i}}^{\prime}=E_{v_{\mathrm{i}}^{\prime} J_{\mathrm{i}}^{\prime}}-E_{v_{\mathrm{i}}^{\prime \prime} J_{\mathrm{i}}^{\prime \prime}}
$$

est, en pratique, calculable avec précision $\left(10^{-3} \mathrm{~cm}^{-1}\right)$ à partir de :

$$
h c v_{0 \mathrm{i}}^{\prime} \# E_{v_{\mathrm{i}}^{\prime}}-E_{v_{\mathrm{i}}^{\prime \prime}}+E_{J_{\mathrm{i}}^{\prime}}-E_{J_{\mathrm{i}}^{\prime \prime}}
$$

où $h$ désigne la constante de Planck et $c$ la célérité du rayonnement dans le vide; $E_{v_{\mathrm{i}}^{\prime} J_{\mathrm{i}}}, E_{v_{v^{\prime \prime}} J_{i}^{\prime \prime}}, E_{v_{1}^{\prime}}, E_{v_{1}^{\prime \prime}}$ représentent les énergies de vibration-rotation et de vibration des niveaux supérieur et inférieur respectivement, rapportées au niveau fondamental; $E_{J^{\prime}}$ et $E_{J^{\prime \prime}}$ sont les énergies de rotation des niveaux supérieur et inférieur de la transition; ces diverses énergies sont exprimées en fonction des données dans le paragraphe 4.
Le coefficient monochromatique d'absorption d'une raie $v_{\mathrm{i}}^{\prime \prime} J_{\mathrm{i}}^{\prime \prime} \rightarrow v_{\mathrm{i}}^{\prime} J_{\mathrm{i}}^{\prime}$ s'écrit :

$$
\kappa_{v_{0 \mathrm{i}}^{\prime}}\left(v^{\prime}\right)=S_{v_{0 \mathrm{i}}^{\prime}} F_{\mathrm{i}}\left(v^{\prime}\right)
$$

$F_{\mathrm{i}}\left(v^{\prime}\right)$ représente le profil normalisé de la raie tel que :

$$
\int_{0}^{\infty} F_{\mathrm{i}}\left(v^{\prime}\right) \mathrm{d} v^{\prime}=1
$$

et $S_{v_{01}^{\prime}}$ désigne l'intensité intégrée de la raie :

$$
S_{v_{01}^{\prime}}=\int_{0}^{\infty} \kappa_{v_{01}^{\prime}} \mathrm{d} v^{\prime}
$$

Le phénomène d'absorption thermique tient compte à la fois de l'absorption vraie et de l'émission induite, caractérisées respectivement par les coefficients d'Einstein $B_{v_{i}^{\prime \prime} J_{i}^{\prime \prime} \rightarrow v_{\mathrm{i}}^{\prime} J_{\mathrm{i}}^{\prime}}$ et $B_{v_{\mathrm{i}}^{\prime} J_{i}^{\prime} \rightarrow v_{\mathrm{i}}^{\prime \prime} J_{i}^{\prime \prime}}$. Ces deux coefficients sont définis par les relations :

$B_{v_{\mathrm{i}}^{\prime \prime} J_{\mathrm{i}}^{\prime \prime} \rightarrow v_{\mathrm{i}}^{\prime} J_{\mathrm{i}}^{\prime}}=8 \pi^{3}\left(3 h^{2}\right)^{-1}\left|\left\langle v_{\mathrm{i}}^{\prime} J_{\mathrm{i}}^{\prime}|\bar{\mu}| v_{\mathrm{i}}^{\prime \prime} J_{\mathrm{i}}^{\prime \prime}\right\rangle\right|^{2}$

et

$$
g_{\mathrm{i}}^{\prime} B_{v_{\mathrm{i}}^{\prime} J_{\mathrm{i}}^{\prime} \rightarrow v_{\mathrm{i}}^{\prime \prime} J_{\mathrm{i}}^{\prime \prime}}=g_{\mathrm{i}}^{\prime \prime} B_{v_{\mathrm{i}}^{\prime \prime} J_{\mathrm{i}}^{\prime \prime} \rightarrow v_{\mathrm{i}}^{\prime} J_{\mathrm{i}}^{\prime}}
$$

où $\left|\left\langle v_{\mathrm{i}}^{\prime} J_{\mathrm{i}}^{\prime}\left|\bar{\mu}_{\mathrm{i}}\right| v_{\mathrm{i}}^{\prime \prime} J_{\mathrm{i}}^{\prime \prime}\right\rangle\right|$ représente l'élément de matrice de l'opérateur dipolaire électrique de la molécule absorbante entre états initial et final $; g_{\mathrm{i}}^{\prime}$ et $g_{\mathrm{i}}^{\prime \prime}$ désignent les dégénérescences des niveaux $v_{\mathrm{i}}^{\prime} J_{\mathrm{i}}^{\prime}$ et $v_{\mathrm{i}}^{\prime \prime} J_{\mathrm{i}}^{\prime \prime}$. Si on considère un flux incident dans un angle solide élémentaire $\mathrm{d} \Omega$, caractérisé par la luminance $L_{v}^{(\text {(I) }}$, la fraction $d^{6} \varphi_{v_{a}^{\prime}}$ de ce flux, absorbée par un élément de volume dư, est donnée par l'expression [9] :

$$
\mathrm{d}^{6} \varphi_{v_{\mathrm{a}}^{\prime}}=\left(B_{v_{\mathrm{i}}^{\prime \prime} J_{\mathrm{i}}^{\prime \prime} \rightarrow v_{\mathrm{i}}^{\prime} J_{\mathrm{i}}^{\prime}} N_{\mathrm{i}}^{\prime \prime}-B_{v_{\mathrm{i}}^{\prime} J_{\mathrm{i}}^{\prime} \rightarrow v_{\mathrm{i}}^{\prime \prime} J_{\mathrm{i}}^{\prime \prime}} N_{\mathrm{i}}^{\prime}\right) F_{\mathrm{i}}\left(v^{\prime}\right) L_{v^{\prime}}^{(\mathrm{I})} h v^{\prime} \mathrm{d} \Omega \mathrm{d} \mathcal{d} v^{\prime}
$$

où $N_{\mathrm{i}}^{\prime \prime}$ et $N_{\mathrm{i}}^{\prime}$ représentent les populations respectives des niveaux $v_{\mathrm{i}}^{\prime \prime} J_{\mathrm{i}}^{\prime \prime}$ et $v_{\mathrm{i}}^{\prime} J_{\mathrm{i}}^{\prime}$. L'identification de (15) à la relation (16) de définition du coefficient monochromatique d'absorption $\kappa_{v_{01}^{\prime}}$ de la raie [1], soit :

$$
\mathrm{d}^{6} \varphi_{v_{\mathrm{a}}^{\prime}}=\kappa_{v_{0 \mathrm{i}}^{\prime}} L_{v^{\prime}}^{(\mathrm{I})} \mathrm{d} \Omega \mathrm{d} U \mathrm{~d} v^{\prime}
$$

conduit à :

$$
\kappa_{v_{01}^{\prime}}=8 \pi^{3}(3 h)^{-1}\left|\left\langle v_{\mathrm{i}}^{\prime} J_{\mathrm{i}}^{\prime}\left|\bar{\mu}_{\mathrm{i}}\right| v_{\mathrm{i}}^{\prime \prime} J_{\mathrm{i}}^{\prime \prime}\right\rangle\right|^{2}\left(N_{\mathrm{i}}^{\prime \prime}-g_{\mathrm{i}}^{\prime \prime} g_{\mathrm{i}}^{\prime-1} N_{\mathrm{i}}^{\prime}\right) F_{\mathrm{i}}\left(v^{\prime}\right) v^{\prime}
$$

L'expression (17), tout à fait générale, est exploitable même si les différents niveaux de vibration évoluent en déséquilibre de population avec les degrés de translation-rotation : $N_{\mathrm{i}}^{\prime \prime}$ et $N_{\mathrm{i}}^{\prime}$ sont alors obtenues à partir de considérations cinétiques : modèles de l'équilibre vibrationnel [10], de la répartition de Treanor [11], équations de relaxation [10], etc...

Nous nous limitons dans cette étude à des mélanges en équilibre thermodynamique local (ETL). Dans ces conditions, la population $N_{\mathrm{i}}$ d'un niveau $v_{\mathrm{i}} J_{\mathrm{i}}$ de dégénérescence $g_{\mathrm{i}}$ est donnée par :

$$
N_{\mathrm{i}}=N x_{\mathrm{i}} g_{\mathrm{i}} \exp \left(-\frac{E_{v_{\mathrm{i}} J_{\mathrm{i}}}}{k T}\right) Q_{\mathrm{i}}^{-1}(T)
$$


où $k$ désigne la constante de Boltzman et $Q_{\mathrm{i}}(T)$ la fonction de partition de vibration-rotation du constituant $\mathrm{i}$, de fraction molaire $x_{\mathrm{i}}$ :

$$
Q_{\mathrm{i}}(T)=\sum_{v_{\mathrm{i}}, J_{\mathrm{i}}} g_{\mathrm{i}} \exp \left(-\frac{E_{v_{\mathrm{i}} J_{\mathrm{i}}}}{k T}\right) .
$$

Compte tenu de (10), (17) et (18), nous obtenons l'expression de l'intensité intégrée de la raie $v_{\mathrm{i}}^{\prime \prime} J_{\mathrm{i}}^{\prime \prime} \rightarrow v_{\mathrm{i}}^{\prime} J_{\mathrm{i}}^{\prime}$ :

$$
S_{\psi_{01}^{\prime}}=\frac{8 \pi^{3}}{3 h} v_{0 \mathrm{i}}^{\prime}\left|\left\langle v_{\mathrm{i}}^{\prime} J_{\mathrm{i}}^{\prime}\left|\bar{\mu}_{\mathrm{i}}\right| v_{\mathrm{i}}^{\prime \prime} J_{\mathrm{i}}^{\prime \prime}\right\rangle\right|^{2} N x_{\mathrm{i}} g_{\mathrm{i}}^{\prime \prime} \exp \left(-\frac{E_{v_{i}^{\prime \prime} J_{\mathrm{i}}^{\prime \prime}}}{k T}\right) Q_{\mathrm{i}}^{-1}\left(1-\exp \left(-\frac{h c v_{0 \mathrm{i}}^{\prime}}{k T}\right)\right) .
$$

Si nous disposions d'une tabulation de l'élément de matrice $\left|\left\langle v_{\mathrm{i}}^{\prime} J_{\mathrm{i}}^{\prime}\left|\bar{\mu}_{\mathrm{i}}\right| v_{\mathrm{i}}^{\prime \prime} J_{\mathrm{i}}^{\prime \prime}\right\rangle\right|$, il serait possible à ce stade de calculer, pour un profil $F_{\mathrm{i}}\left(v^{\prime}\right)$ donné, l'absorptivité monochromatique du mélange gazeux par les relations (2), (3) et (6). Cependant les spectroscopistes se réfèrent à l'intensité intégrée $S_{v_{i}^{\prime \prime} v_{\mathrm{i}}^{\prime}}$ d'une bande d'absorption, définie comme étant la somme des intensités intégrées de toutes les raies la constituant [7], [8] :

$$
S_{v_{\mathrm{i}}^{\prime \prime} v_{\mathrm{i}}^{\prime}}=\sum_{J_{\mathrm{i}}^{\prime}, J_{i}^{\prime \prime}} S_{v_{01}^{\prime}} \text {. }
$$

Comme la bande a une extension faible (de l'ordre de $100 \mathrm{~cm}^{-1}$ ) devant $v_{0}^{\prime}$ et que de plus sa structure est grossièrement symétrique, nous obtenons, en première approximation :

$$
S_{v_{i}^{\prime \prime} v_{i}^{\prime}}=\frac{8 \pi^{3}}{3 h} N x_{\mathrm{i}} v_{\mathrm{B}_{\mathrm{i}}^{\prime}}^{\prime}\left(1-\exp \left(-\frac{h c v_{\mathrm{B}_{\mathrm{i}}^{\prime}}^{\prime}}{k T}\right)\right)\left(\sum_{J_{\mathrm{i}}^{\prime}, J_{\mathrm{i}}^{\prime \prime}} g_{\mathrm{i}}^{\prime \prime} \exp \left(-\frac{E_{v_{i}^{\prime \prime} J_{i}^{\prime \prime}}}{k T}\right)\right) \frac{\left|\left\langle v_{\mathrm{i}}^{\prime} J_{\mathrm{i}}^{\prime}\left|\bar{\mu}_{\mathrm{i}}\right| v_{\mathrm{i}}^{\prime \prime} J_{\mathrm{i}}^{\prime \prime}\right\rangle\right|^{2}}{Q_{\mathrm{i}}(T)}
$$

où $v_{\mathbf{B}_{1}}^{\prime}$, nombre d'onde du centre de bande est défini par :

$$
h c v_{\mathbf{B}_{1}}^{\prime}=E_{v_{\mathrm{i}}^{\prime}}-E_{v_{\mathrm{i}}^{\prime \prime}} \cdot
$$

L'élément de matrice $\left|\left\langle v_{\mathrm{i}}^{\prime} J_{\mathrm{i}}^{\prime}\left|\bar{\mu}_{\mathrm{i}}\right| v_{\mathrm{i}}^{\prime \prime} J_{\mathrm{i}}^{\prime \prime}\right\rangle\right|^{2}$ peut s'exprimer comme le produit de deux facteurs dépendant des coordonnées de vibration et rotation $\left|\left\langle v_{\mathrm{i}}^{\prime}\left|q_{v_{1}}\right| v_{\mathrm{i}}^{\prime \prime}\right\rangle\right|^{2},\left|\left\langle J_{\mathrm{i}}^{\prime}\left|q_{\mathrm{R}_{\mathrm{i}}}\right| J_{\mathrm{i}}^{\prime \prime}\right\rangle\right|^{2}$ et d'un facteur éventuel représentant le couplage rotation-vibration : $(1+m \zeta)^{2}$; par conséquent :

$$
\left|\left\langle v_{\mathrm{i}}^{\prime} J_{\mathrm{i}}^{\prime}\left|\bar{\mu}_{\mathrm{i}}\right| v_{\mathrm{i}}^{\prime \prime} J_{\mathrm{i}}^{\prime \prime}\right\rangle\right|^{2}=(1+m \zeta)^{2}\left|\left\langle v_{\mathrm{i}}^{\prime}\left|q_{v_{\mathrm{i}}}\right| v_{\mathrm{i}}^{\prime \prime}\right\rangle\right|^{2}\left|\left\langle J_{\mathrm{i}}^{\prime}\left|q_{\mathrm{R}_{\mathrm{i}}}\right| J_{\mathrm{i}}^{\prime \prime}\right\rangle\right|^{2}
$$

où $\zeta$ désigne une donnée caractérisant la bande, tandis que $m$ vaut $+J_{i}^{\prime \prime}$ pour une branche $\mathrm{Q}, J_{i}^{\prime \prime}+1$ pour une branche R et $-J_{i}^{\prime \prime}$ pour une branche $P$ [7]. Il est alors commode d'introduire les fonctions de partition vibrationnelle $Q_{v_{1}}$ et rotationnelle $Q_{R_{1}}$ par les relations (25) et (26) qui permettent de les calculer à partir des données $E_{v_{1}}$ et $E_{J_{1}}$ obtenues dans le paragraphe 4 :

$$
\begin{aligned}
& Q_{v_{\mathrm{i}}}(T)=\sum_{v_{\mathrm{i}}} g_{v_{\mathrm{i}}} \exp \left(-\frac{E_{v_{\mathrm{i}}}}{k T}\right) \\
& Q_{\mathrm{R}_{\mathrm{i}}}(T)=\sum_{J_{\mathrm{i}}}\left(2 J_{\mathrm{i}}+1\right) \exp \left(\frac{-E_{J_{\mathrm{i}}}}{k T}\right)
\end{aligned}
$$

où $g_{v_{\mathrm{i}}}$ désigne la dégénérescence vibrationnelle du niveau $v_{\mathrm{i}}$. Nous poserons dans la suite :

$$
L_{\mathrm{R}}\left(J_{\mathrm{i}}^{\prime}, J_{\mathrm{i}}^{\prime \prime}\right)=\left(2 J_{\mathrm{i}}^{\prime \prime}+1\right)\left|\left\langle J_{\mathrm{i}}^{\prime}\left|q_{\mathrm{R}_{\mathrm{i}}}\right| J_{\mathrm{i}}^{\prime \prime}\right\rangle\right|^{2}
$$

$L_{\mathrm{R}}\left(J_{\mathrm{i}}^{\prime}, J_{\mathrm{i}}^{\prime \prime}\right)$, appelé poids statistique d'une raie, vérifie la relation [9] :

$$
\sum_{J_{i}^{\prime}} L_{R}\left(J_{i}^{\prime}, J_{i}^{\prime \prime}\right)=2 J_{i}^{\prime \prime}+1 .
$$

Compte tenu des relations (24) à (28), l'expression (22) de l'intensité intégrée de la bande, à la température $T$ et à la pression totale $P$, devient :

$$
S_{v_{\mathrm{i}}^{\prime \prime} v^{\prime}}=\frac{8 \pi^{3}}{3 h} N x_{\mathrm{i}} v_{\mathrm{B}_{\mathrm{i}}}^{\prime}\left(1-\exp \left(\frac{-h c v_{\mathrm{B}_{\mathrm{i}}}^{\prime}}{k T}\right)\right) Q_{v_{\mathrm{i}}}^{-1}(T) g_{v_{\mathrm{i}}^{\prime \prime}} \exp \left(\frac{-E_{v_{i}^{\prime \prime}}}{k T}\right)\left|\left\langle v_{\mathrm{i}}^{\prime}\left|q_{v_{\mathrm{i}}}\right| v_{\mathrm{i}}^{\prime \prime}\right\rangle\right|^{2} .
$$

La quantité $N^{-1} S_{v^{\prime \prime} v^{\prime}}$ est tabulée pour un constituant pur $\left(x_{\mathrm{i}}=1\right)$, dans les conditions standard $\left(T_{\mathrm{S}}=296 \mathrm{~K}\right.$, 
$\left.P_{\mathrm{S}}=1,013 \times 10^{5} \mathrm{~Pa}\right)$; elle est notée $S_{v_{01}}\left(T_{\mathrm{S}}\right)$. En introduisant cette grandeur, nous obtenons à partir de (20), (22) et (27) l'expression de l'intensité intégrée de la raie $v_{\mathrm{i}}^{\prime \prime} J_{\mathrm{i}}^{\prime \prime} \rightarrow v_{\mathrm{i}}^{\prime} J_{\mathrm{i}}^{\prime}$ :

$$
S_{v_{0 \mathrm{i}}^{\prime}}=x_{\mathrm{i}} \frac{P}{k T} \frac{v_{0 \mathrm{i}}^{\prime}}{v_{\mathrm{B}_{\mathrm{i}}}^{\prime}} S_{v_{01}}\left(T_{\mathrm{s}}\right) \frac{1-\exp \left(-\frac{h c v_{0 \mathrm{i}}^{\prime}}{k T}\right)}{1-\exp \left(-\frac{h c v_{\mathrm{B}_{\mathrm{i}}}^{\prime}}{k T_{\mathrm{S}}}\right)}(1+m \zeta)^{2} \frac{L_{\mathrm{R}_{\mathrm{i}}} \exp \left(-\frac{E_{v_{\mathrm{i}}^{\prime \prime} J_{\mathrm{i}}^{\prime}}}{k T}\right)}{Q_{\mathrm{R}_{\mathrm{i}}}(T) \exp \left(-\frac{E_{v_{i}^{\prime \prime}}}{k T_{\mathrm{S}}}\right)} \frac{Q_{v_{\mathrm{i}}}\left(T_{\mathrm{S}}\right)}{Q_{v_{\mathrm{i}}}(T)}
$$

4. Données spectroscopiques. - Le modèle développé ici utilise les récentes déterminations des intensités intégrées des bandes d'absorption d'un rayonnement par les molécules constituant l'atmosphère terrestre $[7,8]$. D'autre part, il est nécessaire de définir le profil de raie $F_{\mathrm{i}}\left(v^{\prime}\right)$ à prendre en compte. Nous envisagerons d'abord, dans ce paragraphe, les différents profils susceptibles d'être utilisés. Ensuite nous développerons le calcul des énergies et des fonctions de partition et distinguerons les cas où $\mathrm{CO}_{2}$ et une molécule diatomique hétéronucléaire sont les constituants absorbants.

4.1 Profils Des RAIES. - Le profil de raie $F_{\mathrm{i}}\left(v^{\prime}\right)$ dépend essertiellement des conditions de température et de pression et ceci quel que soit le constituant absorbant. Le profil le plus usuel, pour un thermicien, est le profil Lorentzien :

$$
F_{\mathrm{i}}\left(v^{\prime}\right)=\frac{\gamma_{\mathrm{iL}}}{\pi\left[\left(v^{\prime}-v_{0 \mathrm{i}}^{\prime}\right)^{2}+\gamma_{\mathrm{iL}}^{2}\right]}
$$

qui est caractérisé par la largeur à mi-hauteur $\gamma_{\mathrm{iL}}$ dont l'expression est :

$$
\begin{aligned}
& \gamma_{\mathrm{iL}}=\frac{P}{P_{\mathrm{S}}}\left(\sum_{i^{\prime}=1}^{n_{\mathrm{a}}} \gamma_{\mathrm{Sii}^{\prime}}\left(J_{\mathrm{i}}^{\prime \prime}\right)\right. x_{\mathrm{i}^{\prime}}\left(\frac{T_{\mathrm{S}}}{T}\right)^{\beta_{\mathrm{ii}}^{\prime}}+ \\
&\left.+\sum_{j^{\prime}=1}^{n_{\mathrm{i}}} \gamma_{\mathrm{Sij}^{\prime}}\left(J_{\mathrm{i}}^{\prime \prime}\right) y_{\mathrm{j}^{\prime}}\left(\frac{T_{\mathrm{S}}}{T}\right)^{\beta_{\mathrm{ij}^{\prime}}}\right)
\end{aligned}
$$

$x_{\mathrm{i}}^{\prime}$ et $y_{\mathrm{j}^{\prime}}$ désignent les fractions molaires des $n_{\mathrm{a}}$ constituants absorbants et $n_{t}$ constituants transparents, $\gamma_{\mathrm{Sii}^{\prime}}$ et $\gamma_{\mathrm{Sij}^{\prime}}$ les demi-largeurs correspondant à l'élargissement d'une raie du constituant $i$ par les constituants $\mathrm{i}^{\prime}$ et $\mathrm{j}^{\prime} ; \beta_{\mathrm{ii}}$ et $\beta_{\mathrm{ij}}$, sont des paramètres expérimentaux.

L'obtention de $\gamma_{\mathrm{iL}}$ est un des points délicats de la méthode utilisée : de nombreuses déterminations expérimentales ([12] à [17]) ont été réalisées presque exclusivement à des températures inférieures ou égales à $300 \mathrm{~K}$ (voir $\S 4.2$ et 4.3 ). Nous extrapolons ces données par la relation (32) à des températures de l'ordre de $1500 \mathrm{~K}$, ce qui peut engendrer une erreur importante sur $\gamma_{\mathrm{iL}}$. Mais comme nous nous intéressons à un spectre à basse résolution moyenné sur un intervalle spectral $\Delta v^{\prime}$ très grand devant $\gamma_{i L}$, les valeurs de $\alpha_{v^{\prime}}^{\text {br }}$ sont peu sensibles (nous le vérifions d'ailleurs dans le prochain paragraphe) à une variation de $\gamma_{i L}$ de l'ordre de $30 \%$.
A faible pression et température élevée, l'élargissement dominant des raies peut être dû à l'effet Doppler, caractérisé par le profil :

$$
\begin{aligned}
& F_{\mathrm{i}}\left(v^{\prime}\right)=\left(\sqrt{\pi} \gamma_{\mathrm{iD}}\right)^{-1} \sqrt{\log 2} \exp \left(-\left(v^{\prime}-v_{0 \mathrm{i}}^{\prime}\right)^{2} \times\right. \\
&\left.\times(\log 2) \times \gamma_{\mathrm{iD}}^{-2}\right)
\end{aligned}
$$

où $\gamma_{\mathrm{iD}}$ est la demi-largeur Doppler à mi-hauteur :

$$
\gamma_{\mathrm{iD}}=1,13 \times 10^{-8} v^{\prime} \sqrt{T M_{\mathrm{i}}^{-1}}
$$

la masse molaire $M_{\mathrm{i}}$ étant exprimée en $\mathrm{kg}, T$ en $\mathrm{K}$, $\gamma_{\mathrm{iD}}$ et $v^{\prime}$ en $\mathrm{cm}^{-1}$. Dans des conditions où $\gamma_{\mathrm{iL}}$ et $\gamma_{\mathrm{iD}}$ sont de même ordre de grandeur, le profil des raies est plus complexe : on obtient un profil de Voigt, éventuellement perturbé à haute température par l'effet Galatry [18, 19, 20].

4.2 ABSORPTION PAR $\mathrm{CO}_{2}$. - Un certain nombre d'auteurs ont déterminé en fonction de $J_{i}^{\prime \prime}$ les demilargeurs $\gamma_{\mathrm{SCO}_{2}-\mathrm{i}^{\prime}}$, correspondant à l'élargissement d'une raie Lorentzienne de $\mathrm{CO}_{2}$ par un autre constituant absorbant $\left(\mathrm{CO}, \mathrm{CO}_{2} \ldots\right)$, ou $\gamma_{\mathrm{ScO}_{2}-\mathrm{j}^{\prime}}$, correspondant à l'élargissement par un constituant transparent $\left(\mathrm{N}_{2}, \mathrm{O}_{2}, \mathrm{He}, \mathrm{Ne}, \mathrm{Ar}, \mathrm{H}_{2}\right)$, ainsi que $\beta_{\mathrm{ii}}{ }^{\prime}$ et $\beta_{\mathrm{ij}}$ entre 150 et $300 \mathrm{~K}[12,13,17]$. Dans la compilation de l'AFCRL [7], une valeur moyenne de $\gamma_{\mathrm{SCO}_{2}-\mathrm{CO}_{2}}$ égale à $0,07 \mathrm{~cm}^{-1}$, et par conséquent indépendante de $J_{i}^{\prime \prime}$, est préconisée. Nous avons, pour différents spectres relatifs à $\mathrm{CO}_{2}$ pur, calculé $\alpha_{\gamma^{\prime}}^{\text {br à }}$ partir de valeurs de $\gamma_{\mathrm{CO}_{2} \mathrm{~L}}$, déduites par (32), avec $\gamma_{\mathrm{S} \mathrm{CO}_{2}-\mathrm{CO}_{2}}$, soit extrait de la référence [13], soit égal à la valeur $0,07 \mathrm{~cm}^{-1}$. Aucune différence sensible n'a été mise en évidence (voir Fig. 7 du $\S 5$ ). En conséquence nous avons adopté, dans les autres calculs, la valeur :

$$
\gamma_{\mathrm{SCO}_{2}-\mathrm{CO}_{2}}=0,07 \mathrm{~cm}^{-1}
$$

et les valeurs du rapport $\gamma_{\mathrm{SCO}_{2}-\mathrm{i}^{\prime}} \gamma_{\mathrm{S} \mathrm{O}_{2}-\mathrm{CO}_{2}}$, préconisées dans [12], soit $0,83,0,65,0,49,0,68,1,17$, quand $i^{\prime}$ désigne respectivement $\mathrm{N}_{2}, \mathrm{Ar}, \mathrm{He}, \mathrm{O}_{2}, \mathrm{H}_{2}$. Pour tous les couples gazeux considérés, le paramètre $\beta_{\mathrm{CO}_{2}-\mathrm{i}^{\prime}}$ a été choisi égal à [7] :

$$
\beta_{\mathrm{CO}_{2}-\mathrm{i}^{\prime}}=0,5 .
$$

Il faut toutefois noter que cette détermination n'a été réalisée qu'au voisinage de la température ambiante et qu'on ne dispose pas de données pour des températures dépassant $370 \mathrm{~K}$. Nous avons donc extrapolé 
cette loi à toutes températures. Selon d'autres déterminations à température inférieure à $370 \mathrm{~K}$, dues à Planet et Tettemer [17] notamment, $\beta$ dépendrait de la nature de la bande, voire de la raie considérée : on trouve les valeurs de $\beta_{\mathrm{CO}_{2}-\mathrm{i}}$ égales à 0,7 [13], 0,852 et 0,948 [17]. L'extrapolation de ces résultats à $1500 \mathrm{~K}$ est discutée dans le paragraphe 5 .

Un phénomène d'origine complexe modifie, dans le cas de $\mathrm{CO}_{2}$, le profil d'une raie d'absorption Lorentzienne très intense, pour des valeurs de $\left|v^{\prime}-v_{0 \mathrm{i}}^{\prime}\right|$ élevées (supérieures à $5 \mathrm{~cm}^{-1}$ ). La raie s'atténue alors exponentiellement, selon des corrélations expérimentales. Nous adopterons l'expression (31) du profil de raie si $\left|v^{\prime}-v_{0 \mathrm{i}}^{\prime}\right|$ est inférieur à $5 \mathrm{~cm}^{-1}$ et l'expression (37) ci-dessous [21] :

$$
\begin{aligned}
F_{\mathrm{i}}\left(v^{\prime}\right)=\gamma_{\mathrm{iL}} & \pi^{-1}\left(\gamma_{\mathrm{iL}}^{2}+\left(v^{\prime}-v_{0 \mathrm{i}}^{\prime}\right)^{2}\right)^{-1} \times \\
& \times \exp \left(-0,601\left[\left|v^{\prime}-v_{0 \mathrm{i}}^{\prime}\right|-5\right]^{0,434}\right)
\end{aligned}
$$

si la quantité $\left|v^{\prime}-v_{0 \mathrm{i}}^{\prime}\right|$ est supérieure à $5 \mathrm{~cm}^{-1}$.

Examinons maintenant quelles sont les autres données nécessaires au calcul de (30) :

- La quantité $S_{v_{0}}\left(T_{\mathrm{s}}\right)$ est tabulée pour toutes les bandes considérées, de même que le nombre d'onde du centre de bande $v_{\mathrm{B}}^{\prime}$ et le paramètre $\zeta[7,8]$.

- Dans les mêmes tables, on trouve les énergies $E_{v}$ des différents niveaux vibrationnels de $\mathrm{CO}_{2}$ notés $v_{1} v_{2}^{l} v_{3}$ où $v_{1}, v_{2}, v_{3}$ représentent respectivement le nombre de quanta dans les modes symétrique, transverse et antisymétrique et $l$ le module de la projection sur l'axe de la molécule du moment cinétique vibratoire.

- La fonction de partition de vibration $Q_{v}(T)$ a été calculée de deux façons. La première méthode consiste à utiliser l'expression (25) à partir des valeurs de $E_{v_{1}}$ des références [7] et [8] (pour des valeurs de $E_{v_{1}}$ allant jusqu'à $9000 \mathrm{~cm}^{-1}$ environ). Dans la deuxième méthode, on adopte les données de la référence [28] relatives à $E_{v_{1}}$ et tenant compte de l'anharmonicité. Aucune différence notable n'apparait entre ces deux méthodes en dessous de $1700 \mathrm{~K}$. Au-dessus de $1700 \mathrm{~K}$, la seconde méthode est obligatoire.

- Pour un niveau de vibration donné $v$, l'énergie de rotation associée à la valeur $J$ du nombre quantique s'écrit :

$$
E_{J}=B_{v}\left[J(J+1)-l^{2}\right]-D_{v}\left[J(J+1)-l^{2}\right]^{2} .
$$

Les constantes $B_{v}$ et $D_{v}$ sont données pour chaque niveau $v$ ([7] et [8]). Il faut noter que le niveau de vibration est dégénéré, si $l$ est différent de 0 , en deux états notés $\mathrm{c}$ et $\mathrm{d}$. A ces états sont associés des couples de valeurs $B_{v_{\mathrm{c}}}, D_{v_{\mathrm{c}}}$ et $B_{v_{\mathrm{d}}}, D_{v_{\mathrm{d}}}$ différentes. Par suite de considérations de symétrie, seuls existent les niveaux rotationnels correspondant, si la quantité $v_{2}+v_{3}$ est paire, à des valeurs de $J$ paires pour un niveau c et impaires pour un niveau d, ou l'inverse, si la quantité $v_{2}+v_{3}$ est impaire [9]. Les valeurs de $L_{\mathrm{R}}$ à prendre en compte sont données dans les références [9] et [7].

Pour une transition entre un niveau $v_{1}^{\prime \prime} v_{2}^{\prime \prime \prime} v_{3}^{\prime \prime}$ et un niveau $v_{1}^{\prime} v_{2}^{\prime \prime} v_{3}^{\prime}$, l'organigramme de la génération des raies est donné sur la figure 3 . La fonction de partition de rotation $Q_{\mathrm{R}}(T)$ est calculée directement à partir de (26) et de (38) pour chaque température.

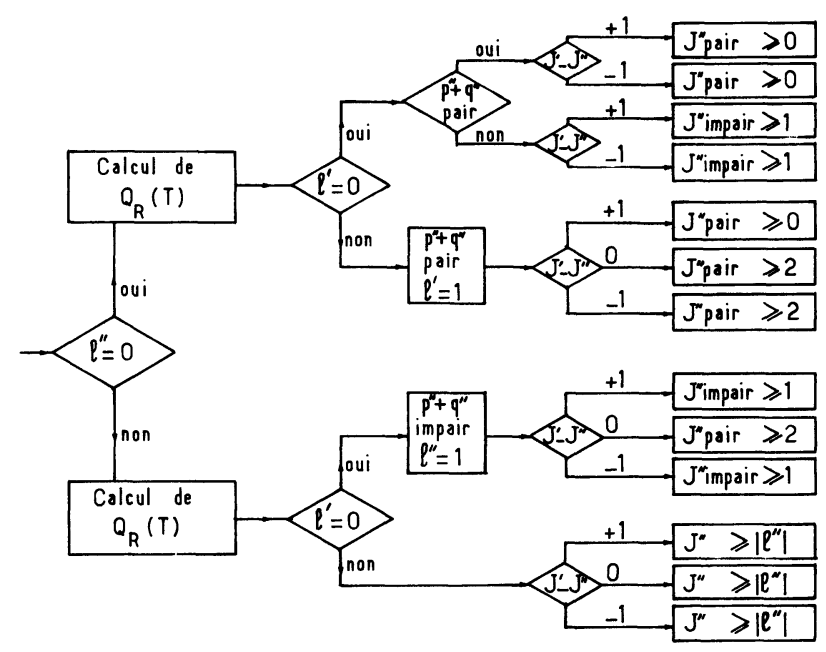

Fig. 3. - Organigramme de la génération des raies d'absorption de $\mathrm{CO}_{2}$ pour la bande $: v_{1}^{\prime \prime} v_{2}^{\prime \prime \prime} v_{3}^{\prime \prime} \rightarrow v_{1}^{\prime} v_{2}^{\prime l^{\prime}} v_{3}^{\prime}$

[Organigram to generate the absorption lines of the $\mathrm{CO}_{2}$ band : $v_{1}^{\prime \prime} v_{2}^{\prime \prime l^{\prime}} v_{3}^{\prime \prime} \rightarrow v_{1}^{\prime} v_{2}^{\prime \prime^{\prime}} v_{3}^{\prime \prime}$.]

\section{3 ABSORPTION PAR UNE MOLÉCULE DIATOMIQUE. -} Le cas de l'absorption par une molécule diatomique hétéronucléaire (par exemple $\mathrm{CO}$ ) est beaucoup plus simple que celui de $\mathrm{CO}_{2}$. Passons rapidement en revue les choix effectués :

- En ce qui concerne les raies d'absorption, de type Lorentzien, les demi-largeurs $\gamma_{\mathrm{CO}-\mathrm{co}}$ et $\gamma_{\mathrm{CO}-\mathrm{i}^{\prime}}$ où i' désigne un autre constituant $\left(\mathrm{N}_{2}, \mathrm{O}_{2}, \mathrm{H}_{2}, \mathrm{HCl}, \mathrm{NO}\right.$ et $\mathrm{CO}_{2}$ ) ont été déterminées de 150 à $300 \mathrm{~K}$ par différents auteurs $[14,15]$ et même de 150 à $800 \mathrm{~K}[16]$. Dans la compilation de l'AFCRL [7], la valeur préconisée pour l'auto-élargissement par CO est :

$$
\gamma_{\mathrm{s} \mathrm{CO}-\mathrm{co}}=0,06 \mathrm{~cm}^{-1} \text {. }
$$

Nous avons adopté cette valeur dans nos calculs et les valeurs du rapport $\gamma_{\mathrm{SCO}-\mathrm{i}^{\prime}} \gamma_{\mathrm{S} \text { Co-co }}^{-1}$ données, pour la valeur 15 de $J^{\prime \prime}$, dans [15]; soit $1,07,0,91,0,81 \mathrm{si}$ $i^{\prime}$ désignent respectivement $\mathrm{CO}_{2}, \mathrm{~N}_{2}$ ou $\mathrm{O}_{2}$. Quel que soit le partenaire de collision, nous avons posé pour le paramètre $\beta_{\mathrm{CO}-\mathrm{i}^{\prime}}$, défini en $(32)[15,16]$ :

$$
\beta_{\mathrm{CO}-\mathrm{i}^{\prime}}=0,75 .
$$

- Les quantités $S_{v_{0}}\left(T_{\mathrm{s}}\right)$ ainsi que les énergies des bandes sont tabulées comme dans le cas de $\mathrm{CO}_{2}$.

- Les niveaux de vibration ne sont pas dégénérés; il n'existe que deux branches par bandes : 
- la branche $\mathrm{R}$, pour laquelle nous avons :

$$
L_{\mathrm{R}}\left(J_{i}^{\prime}=J_{i}^{\prime \prime}+1, J_{i}^{\prime \prime}\right)=J_{i}^{\prime \prime}+1
$$

- la branche $P$ telle que :

$$
L_{\mathrm{R}}\left(J_{i}^{\prime}=J_{i}^{\prime \prime}-1, J_{i}^{\prime \prime}\right)=J_{i}^{\prime \prime} .
$$

5. Résultats et discussions. - Nous disposons d'un nombre limité de spectres expérimentaux à températures et pressions élevées. Dans le cas de $\mathrm{CO}_{2}$ pur, il faut citer notamment ceux d'Edwards [2], de Ben Arieh [4], Gryvnak et Burch [25] cités par Oppenheim et Ben Arieh [4, 22]; les gammes de température et de pression couvertes vont de 800 à $1500 \mathrm{~K}$ et de $1,5 \times 10^{4} \mathrm{~Pa}$ à $10^{6} \mathrm{~Pa}$. Pour le mélange $\mathrm{CO}_{2}-\mathrm{N}_{2}$, nous avons utilisé comme référence le spectre de Gryvnak et Burch [25] à $1500 \mathrm{~K}$ et $10^{5} \mathrm{~Pa}$. Ces spectres ont été obtenus avec une précision acceptable de 5 à $10 \%$, une résolution variable de quelques $\mathrm{cm}^{-1}$ à $175 \mathrm{~cm}^{-1}$, dans des conditions bien déterminées de température et de pression, pour lesquelles on peut admettre l'hypothèse de l'équilibre thermodynamique local (ETL).

Nous comparons à ces spectres expérimentaux ceux établis avec la méthode exposée dans les paragraphes 2 et 3 , qui utilise un calcul raie par raie, fondé sur.les données spectroscopiques $[7,8]$ et sur les approximations développées dans le paragraphe 2 . Nous évoquerons également le cas de spectres obtenus au sein d'une flamme, à $3000 \mathrm{~K}$ environ, [23]. Enfin nous avons calculé le spectre d'un mélange ternaire $\mathrm{CO}_{2}-\mathrm{CO}-\mathrm{N}_{2}$ à $1500 \mathrm{~K}$ et $10^{5} \mathrm{~Pa}$, sans disposer malheureusement de spectre expérimental de référence.

Tous ces spectres ont été obtenus avec une valeur des paramètres $\beta_{\mathrm{CO}_{2}-\mathrm{i}}$ égale à 0,5 [7]; il faut cependant signaler que certains auteurs préconisent, à température beaucoup plus modérée (inférieure à $350 \mathrm{~K}$ ), d'autres valeurs : une discussion de ce point est nécessaire. Comme la valeur de $\beta_{\mathrm{CO}_{2-\mathrm{i}}}$ est d'autant plus critique que la température est élevée, nous mènerons cette discussion à propos d'un spectre à $1500 \mathrm{~K}$. L'influence de l'évolution de la largeur de raie $\gamma_{\mathrm{CO}_{2}-\mathrm{i}}$ avec le nombre quantique de rotation est d'autre part évoquée à propos d'un autre spectre à $833 \mathrm{~K}$.

Le premier spectre étudié, dû à Burch et Gryvnak [25], représente (Fig. 4) l'émissivité d'une colonne de $\mathrm{CO}_{2}$ pur, de $0,0775 \mathrm{~m}$ de long, à $1500 \mathrm{~K}$, sous une pression de $0,26 \times 10^{5} \mathrm{~Pa}$, dans l'intervalle [2 100, $2400 \mathrm{~cm}^{-1}$ ] : il a été obtenu avec une résolution de l'ordre $\mathrm{du} \mathrm{cm}^{-1}$, voisine de celle de notre calcul. Les spectres expérimentaux et théoriques sont donc comparables directement. Le calcul a été conduit tous les $20 \mathrm{~cm}^{-1}$; l'intégration numérique a été réalisée en chaque point sur une largeur de $1 \mathrm{~cm}^{-1}$ avec 100 puis 200 pas d'intégration. La durée de ce calcul varie suivant la valeur de $\beta_{\mathrm{CO}_{2}-\mathrm{i}}$ et le nombre de pas : elle est de l'ordre de la minute sur un ordinateur IBM 370, avec 100 pas d'intégration. Le spectre expérimental en trait plein et le spectre calculé avec $\beta$ égal à 0,5 ,

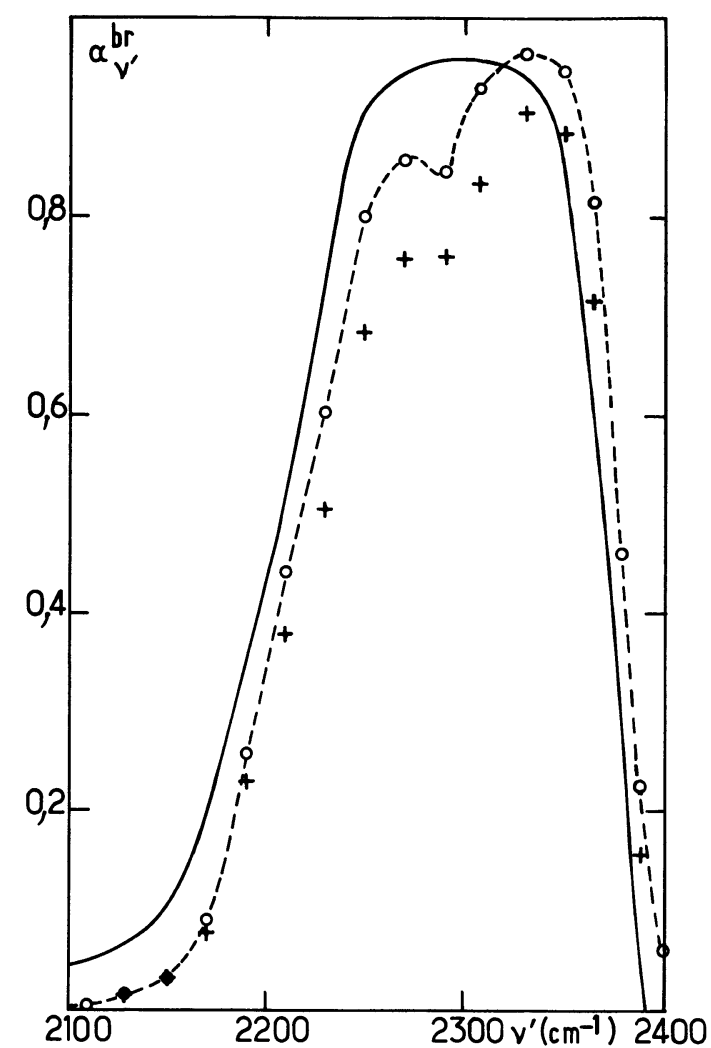

Fig. 4. - Spectres d'absorption de $\mathrm{CO}_{2}: P=2,60 \times 10^{4} \mathrm{~Pa}$; $d=0,0775 \mathrm{~m} ; x_{\mathrm{CO}_{2}}=1 ; T=1500 \mathrm{~K}$. - : spectre expérimental [25]. Tous les calculs sont faits avec $\gamma_{\mathrm{SCO}_{2}-\mathrm{CO}_{2}}=0,07 \mathrm{~cm}^{-1}$. --- : spectre et $O$ : points calculés avec $\beta_{\mathrm{CO}_{2}-\mathrm{CO}_{2}}=0,5$. + : points calculés avec $\beta_{\mathrm{CO}_{2}-\mathrm{CO}_{2}}=0,95$.

en pointillés (passant par les points de calcul équidistants de $20 \mathrm{~cm}^{-1}$ ), concordent sinon parfaitement $\mathrm{du}$ moins suffisamment pour un thermicien. Nous avons aussi réalisé le calcul pour différentes valeurs de $\beta$; les résultats obtenus pour $\beta=0,95$, valeur voisine de la valeur extrême obtenue par Tettemer et Planet [17], correspondent aux croix de la figure 4. L'accord avec les mesures devient alors médiocre. Les spectres correspondants aux valeurs 0,7 , ou 0,85 de $\beta$, non représentés sur la figure 4 , sont intermédiaires entre la courbe en pointillés et une courbe passant par les croix. Nous avons donc retenu la valeur préconisée dans la référence [7] soit $\beta=0,5$ pour tous les spectres ultérieurs qui correspondent à des températures élevées.

Un deuxième spectre d'émission dans l'intervalle [2 100, $.2400 \mathrm{~cm}^{-1}$ ] pour un mélange $\mathrm{CO}_{2}-\mathrm{N}_{2}$ de fraction molaire $x_{\mathrm{CO}_{2}}$ égale à 0,145 a été obtenu à $1500 \mathrm{~K}$ et $1,02 \times 10^{5} \mathrm{~Pa}$ par Burch et Gryvnak [25] avec une résolution de l'ordre $\mathrm{du} \mathrm{cm}^{-1}$ pour une longueur de cellule de $0,0775 \mathrm{~m}$. Il est comparé sur la figure 5 avec nos calculs, réalisés dans des conditions et des temps similaires à ceux du spectre précédent, à partir des données définies dans, le paragraphe 4. Nous avons négligé dans le calcul la présence éventuelle d'un continuum d'absorption induit par collisions; cette hypothèse a d'une manière 


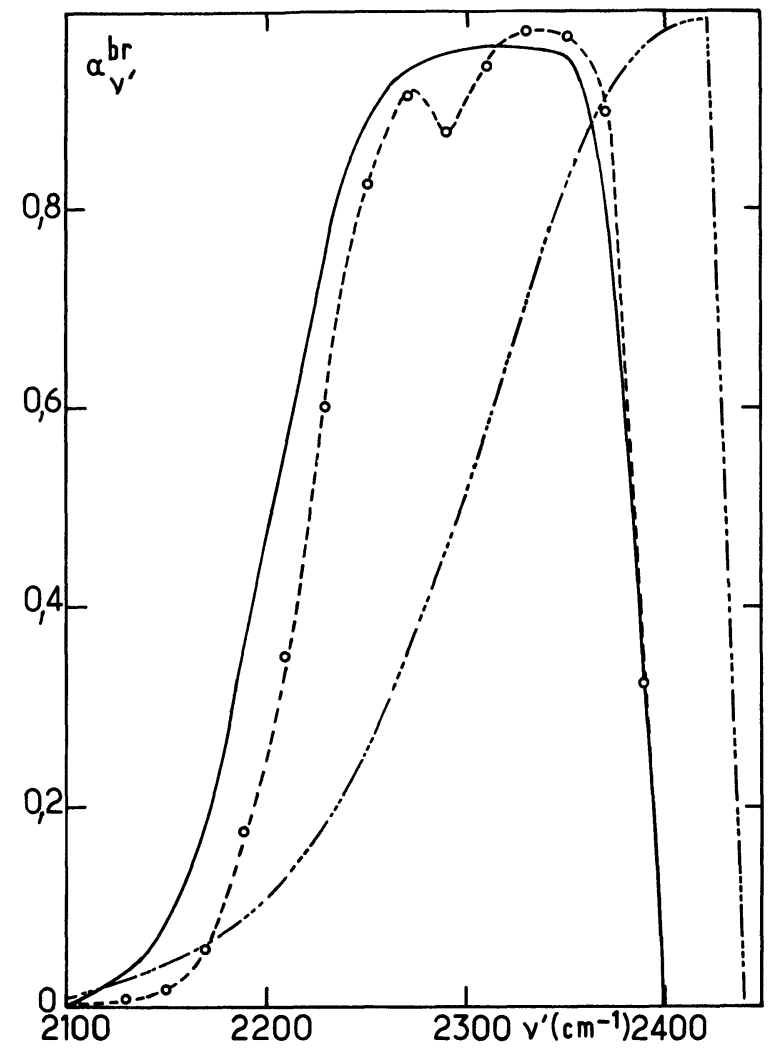

Fig. 5. - Spectres d'absorption pour un mélange $\mathrm{CO}_{2}-\mathrm{N}_{2}$ : $P=1,02 \times 10^{5} \mathrm{~Pa} ; d=0,0775 \mathrm{~m} ; x_{\mathrm{CO}_{2}}=0,145 ; x_{\mathrm{N}_{2}}=0,855$; $T=1500 \mathrm{~K}$. $:$ spectre expérimental [25]; - spectre et $O$ : points calculés avec $\gamma_{\mathrm{CO}_{2}-\mathrm{CO}_{2}}=0,07 \mathrm{~cm}^{-1}$ et $\beta_{\mathrm{CO}_{2-1}}=0,5 ;-\ldots$ : calcul réalisé dans la référence [26], à partir de la méthode indiquée dans la référence [2].

générale été faite par tous les auteurs cités $[2,4,5]$. L'accord entre spectres théorique et expérimental est satisfaisant. Sur la figure 5 a été également représenté en traits pointillés mixtes le spectre obtenu par Paramo [21] en utilisant le modèle d'Edwards [2]. Si la surface de la bande noire équivalente (largeur équivalente) est satisfaisante, la position et le profil de la bande d'absorption ainsi obtenus sont largement erronés.

Un autre spectre d'absorption par $\mathrm{CO}_{2}$ pur, à $1200 \mathrm{~K}$ et $1,58 \times 10^{4} \mathrm{~Pa}$, pour une longueur de $0,1505 \mathrm{~m}$ [4], est représenté sur la figure 6 et comparé au spectre que nous avons calculé, de la même façon que les précédents, et à celui calculé par Bernstein [6] à partir d'un modèle de bandes : les écarts entre le spectre expérimental, dont la précision est de l'ordre de 5 à $10 \%$, et les deux spectres calculés sont du même ordre.

Un spectre d'absorption pour un mélange ternaire $\mathrm{CO}_{2}-\mathrm{CO}-\mathrm{N}_{2}$, caractérisé par les fractions molaires $x_{\mathrm{CO}_{2}}=0,15, x_{\mathrm{CO}}=0,35$ a été calculé pour une longueur $d$ de $0,1 \mathrm{~m}$ à $1500 \mathrm{~K}$ et $10^{5} \mathrm{~Pa}$. Il est représenté sur la figure 7 . Il n'existe pas de spectre expérimental de référence dans ces conditions.

Nous ne disposons pas de spectres à température supérieure à $1500 \mathrm{~K}$, à l'exception de ceux relevés

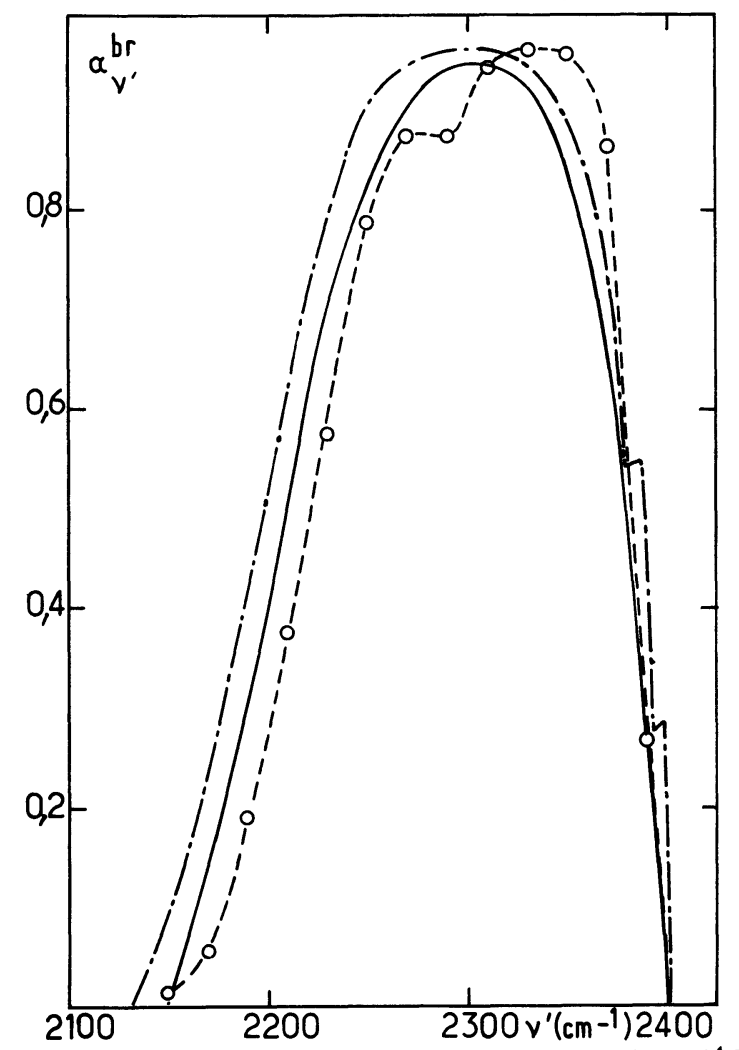

Fig. 6. - Spectres d'absorption de $\mathrm{CO}_{2}: P=1,58 \times 10^{4} \mathrm{~Pa}$, $d=0,1505 \mathrm{~m}, x_{\mathrm{CO}_{2}}=1, T=1200 \mathrm{~K}$. rimental [4]; - - : spectre et $O$ : points calculés avec $\gamma_{\mathrm{CO}_{2}-\mathrm{CO}_{2}}=0,07 \mathrm{~cm}^{-1}$ et $\beta_{\mathrm{CO}_{2}-\mathrm{CO}_{2}}=0,5 ;--\ldots$ : spectre calculé par [6].

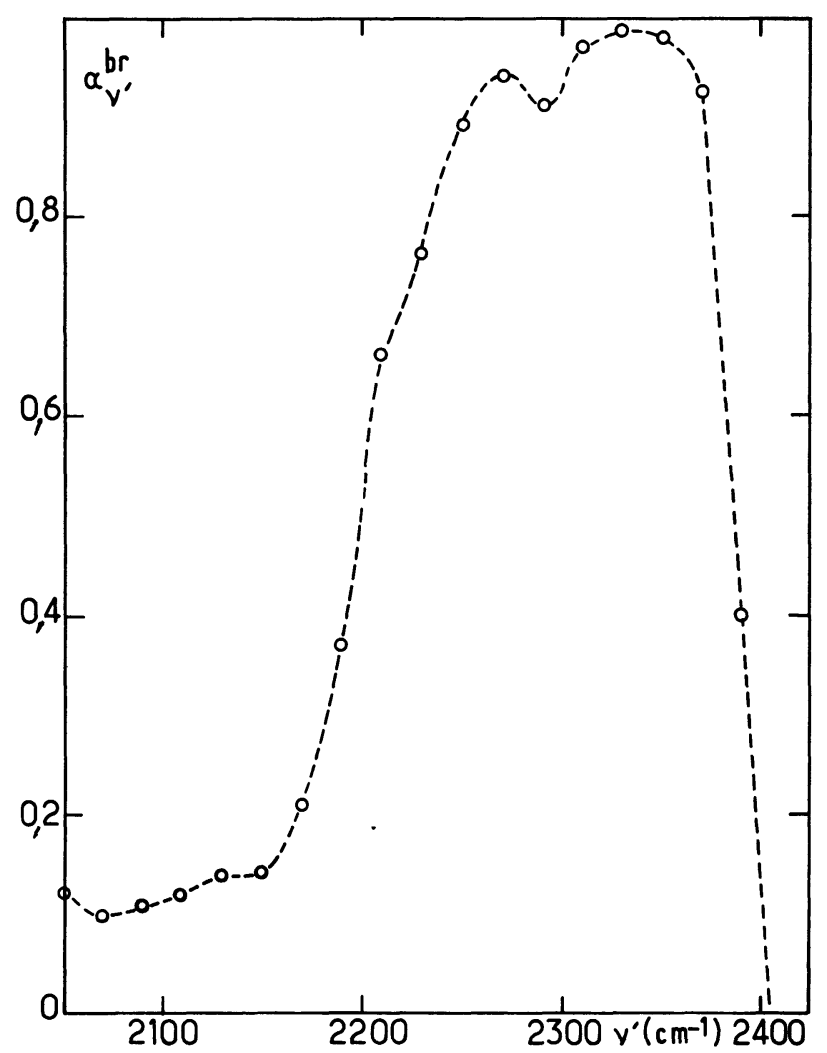

Fig. 7. - Spectre d'absorption pour un mélange $\mathrm{CO}_{2}-\mathrm{N}_{2}-\mathrm{CO}$; $P=10^{5} \mathrm{~Pa}, d=0,10 \mathrm{~m}, x_{\mathrm{CO}_{2}}=0,150, x_{\mathrm{N}_{2}}=0,500, x_{\mathrm{Co}}=0,350$, $T=1500 \mathrm{~K}$; - - : spectre et $O$ : points calculés avec $\beta_{\mathrm{CO}_{2}-\mathrm{i}}=0,5$ et $\gamma_{\mathrm{CO}_{2}-\mathrm{CO}_{2}}=0,07 \mathrm{~cm}^{-1}$. 
par Ludwig et Ferriso [23] à travers une flamme de combustion de kérosène, qui comporte donc de nombreuses impuretés et molécules non identifiées. La température $T$, supposée constante dans la flamme, et les fractions molaires en quelques constituants principaux ont été déduites de mesures pyrométriques, sans qu'on connaisse bien l'incertitude sur ces divers paramètres. Ces raisons contribuent à expliquer l'impossibilité d'une interprétation correcte de ce spectre dans tout l'intervalle spectral, en particulier vers les faibles nombres d'onde. D'autres explications sont également envisageables : l'équilibre de population entre les différents niveaux de vibration rotation n'est pas assuré, de manière évidente, au sein d'une flamme à $3000 \mathrm{~K}$. Enfin, il est possible qu'il manque, dans les compilations [7] et [8], des bandes très chaudes, importantes à $3000 \mathrm{~K}$ et négligeables au voisinage de $300 \mathrm{~K}$

Les derniers spectres que nous envisageons, dus à Edwards [2, 27], sont relatifs à l'absorption par une colonne gazeuse de $\mathrm{CO}_{2}$, longue de $0,388 \mathrm{~m}$, à $10^{6} \mathrm{~Pa}$ et $833 \mathrm{~K}$; dans ces conditions, trois intervalles spectraux ont été utilisés, centrés en 700,2300 et $3600 \mathrm{~cm}^{-1}$. Les spectres correspondants sont représentés en traits pleins figures 8,9 et 10 . Il faut noter que la résolution du spectromètre, indiquée dans la référence [2], est relativement médiocre : $33 \mathrm{~cm}^{-1}, 61 \mathrm{~cm}^{-1}$ et $175 \mathrm{~cm}^{-1}$ respectivement, pour les trois intervalles spectraux cités précédemment. Elle est très inférieure à celle du calcul de $\alpha_{v^{\prime}}^{\text {br }}\left(\right.$ de $\left.5 \mathrm{~cm}^{-1}\right)$.

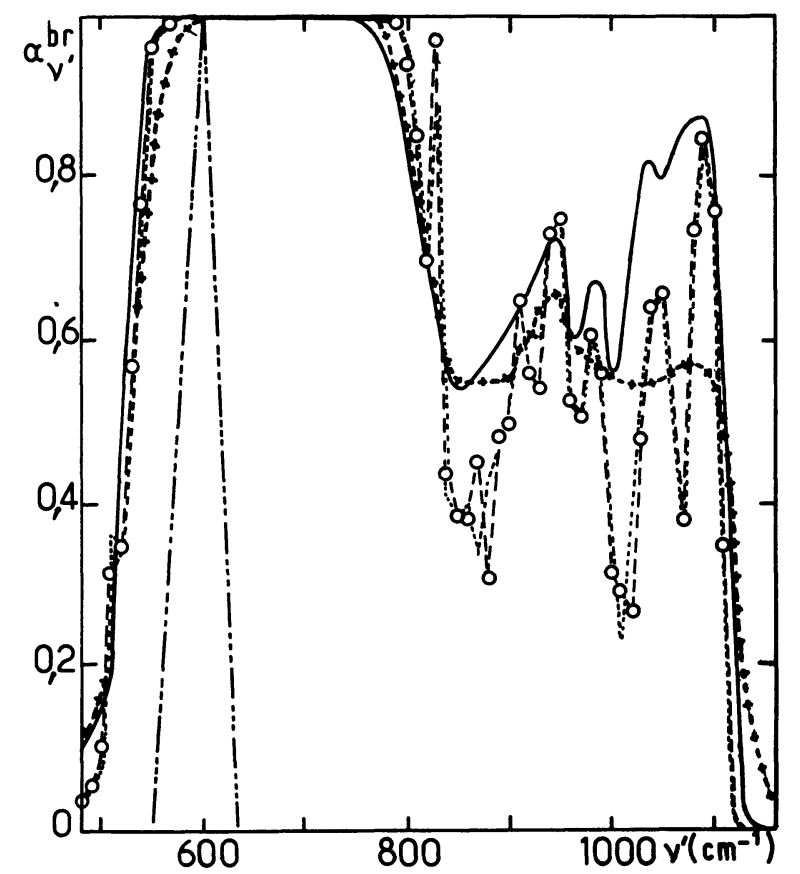

Fig. 8. - Spectres d'absorption de $\mathrm{CO}_{2} ; P=10^{6} \mathrm{~Pa}, d=0,388 \mathrm{~m}$, $x_{\mathrm{CO}_{2}}=1, \mathrm{~T}=833 \mathrm{~K} ;-$ : spectre expérimental $;---$ : spectre et $O$ : points calculés avec $\gamma_{\mathrm{SCO}_{2}}=0,07 \mathrm{~cm}^{-1}$ et $\beta_{\mathrm{CO}_{2}-\mathrm{CO}_{2}}=0,5 ;-\ldots-$ : spectre calculé avec $\gamma_{\mathrm{SCO}_{2}-\mathrm{CO}_{2}}$ donné par (37) et $\beta_{\mathrm{CO}_{2}-\mathrm{CO}_{2}}=0,5 ;-\ldots$ : fonction d'appareil approximative du spectromètre utilisé par [2]; +-+-+- : spectre obtenu par convolution du spectre calculé par la fonction d'appareil.

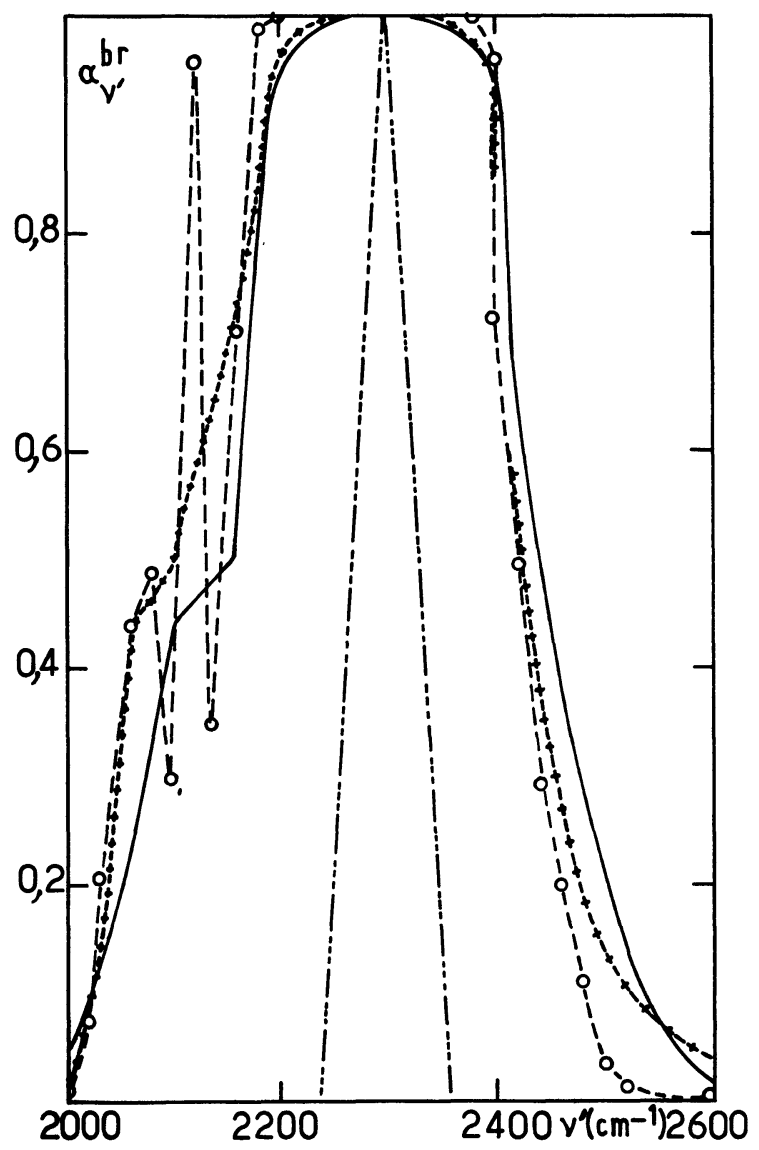

Fig. 9. - Spectres d'absorption de $\mathrm{CO}_{2} ; P=10^{6} \mathrm{~Pa}, d=0,388 \mathrm{~m}$, $x_{\mathrm{CO}_{2}}=1, T=833 \mathrm{~K}$. - : spectre expérimental; - -

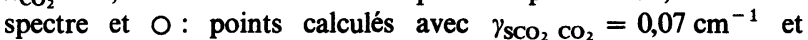
$\beta_{\mathrm{CO}_{2}-\mathrm{CO}_{2}}=0,5 ;-$ : fonction d'appareil approximative $\mathrm{du}$ spectromètre utilisé par [2]; +-+-+- : spectre obtenu par convolution du spectre calculé par la fonction d'appareil.

Nous avons utilisé pour déterminer $\alpha_{v^{\prime}}^{\text {br }} 53$ bandes d'absorption $v^{\prime \prime} \rightarrow v^{\prime}$ de $\mathrm{CO}_{2}$ pour l'intervalle spectral centré en $700 \mathrm{~cm}^{-1}$ (de $v_{\mathrm{B}}^{\prime}=508,141 \mathrm{~cm}^{-1}$ à $\left.v_{\mathrm{B}}^{\prime}=1074,271 \mathrm{~cm}^{-1}\right), 85$ bandes pour l'intervalle centré en $2300 \mathrm{~cm}^{-1}$ (de $v_{\mathrm{B}}^{\prime}=2076,865 \mathrm{~cm}^{-1}$ à $v_{\mathrm{B}}^{\prime}=2458,158 \mathrm{~cm}^{-1}$ ) et 35 bandes pour l'intervalle centré en $3600 \mathrm{~cm}^{-1}$ (de $v_{\mathrm{B}}^{\prime}=3504,933 \mathrm{~cm}^{-1}$ à $v_{\mathrm{B}}^{\prime}=3858,109 \mathrm{~cm}^{-1}$ ). Les évolutions de $\alpha_{v^{\prime}}^{\mathrm{br}}$, en fonction de $v^{\prime}$ sont représentées sur les figures 8,9 et 10 en traits pointillés. Le calcul a été réalisé de $20 \mathrm{~cm}^{-1}$ en $20 \mathrm{~cm}^{-1}$ et la moyenne, donnée par l'expression (3), a été faite sur un intervalle

$$
\Delta v^{\prime}=5 \mathrm{~cm}^{-1} \text {. }
$$

Le nombre de raies, contribuant de façon appréciable à la valeur de $\alpha_{v^{\prime}}^{\text {br }}$, varie suivant la région du spectre considérée entre quelques centaines et quelques milliers. Compte tenu de la résolution médiocre des résultats expérimentaux, nous avons calculé un spectre approximatif en convoluant le spectre que nous avons obtenu avec une fonction d'appareil très approchée, correspondant aux conditions expérimentales.

Nous avons également comparé les résultats obtenus pour $\alpha_{v^{\prime}}^{\text {br }}$ en adoptant successivement la valeur de 


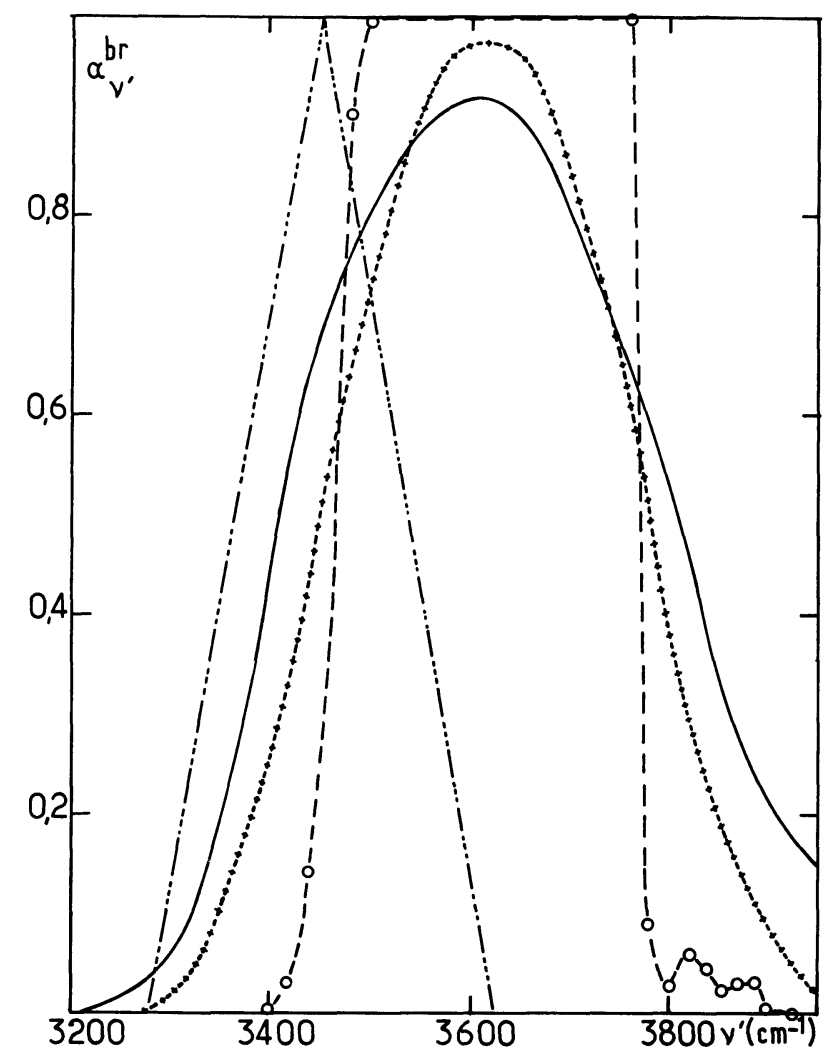

Fig. 10. - Spectres d'absorption de $\mathrm{CO}_{2} ; P=10^{6} \mathrm{~Pa}, d=0,388 \mathrm{~m}$, $x_{\mathrm{CO}_{2}}=1, T=833 \mathrm{~K}$. - : spectre expérimental; -.-.-.-.. :

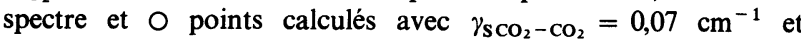
$\beta_{\mathrm{CO}_{2}-\mathrm{CO}_{2}}=0,5 ;-\ldots$ : fonction d'appareil approximative $\mathrm{du}$ spectromètre utilisé par [2]; +-+-+- : spectre obtenu par convolution du spectre calculé par la fonctinn d'appareil.

$\gamma_{\mathrm{SCO}_{2}-\mathrm{CO}_{2}}$, égale à $0,07 \mathrm{~cm}^{-1}$, indépendante de $J^{\prime \prime}$, préconisée dans [7] et une expression, fonction de $J^{\prime \prime}$, tirée des résultats de la référence [20] :

$$
\begin{aligned}
0 \leqslant J^{\prime \prime} & \leqslant 60 \gamma_{\mathrm{SCO}_{2}-\mathrm{CO}_{2}}=0,12\left(1-0,0128 J^{\prime \prime}\right) \mathrm{cm}^{-1} \\
J^{\prime \prime} & \geqslant 60 \gamma_{\mathrm{SCO}_{2}-\mathrm{CO}_{2}}=0,03 \mathrm{~cm}^{-1} .
\end{aligned}
$$

Les deux courbes $\alpha_{v^{\prime}}^{\text {br }}$ correspondantes sont représentées figure 8 pour l'intervalle spectral centré en
$700 \mathrm{~cm}^{-1}$. Aucune différence significative n'apparaît entre ces deux résultats dans ce cas, ni pour les autres intervalles spectraux considérés.

Les temps de calcul correspondant à ces trois spectres sont un peu plus importants que les précédents, car le nombre de raies actives est plus important : 3 min sur IBM 370. Ils ont cependant été minimisés, le calcul ayant été arrêté dès que l'absorptivité dépassait 0,999 dans certaines régions spectrales.

6. Conclusion. - Nous avons confronté la méthode de calcul, développée dans cet article, à un certain nombre de spectres expérimentaux, à pression et température élevées, dans des conditions où l'hypothèse de l'équilibre local est vérifiée, au moins approximativement. L'accord est satisfaisant pour une gamme très étendue du produit $P \mathrm{dx}_{\mathrm{CO}_{2}}$; par ailleurs, la méthode de calcul n'est pas limitée à un profil de raie particulier (Lorentz, Doppler, Voigt...). Le programme que nous avons mis au point permet actuellement :

- de calculer le spectre d'absorption d'un mélange gazeux de $\mathrm{CO}_{2}$ avec un gaz diatomique absorbant et un gaz inerte quelconque. Le calcul est généralisable au cas où les degrés de liberté des molécules, translation, rotation et vibration, évoluent en déséquilibre;

- de déterminer les valeurs des coefficients d'absorption monochromatiques, nécessaires à la résolution de l'équation de transfert par rayonnement, dans certains types de problèmes.

Le programme, qui doit encore être optimisé de façon à abaisser les temps de calcul pour des applications de laboratoire ou industrielles, peut être mis à la disposition de personnes intéressées. Nous envisageons d'y inclure, dans un proche avenir, l'absorption par $\mathrm{H}_{2} \mathrm{O}$.

Remerciements. - Les auteurs tiennent à remercier Mme Magdeleine Huetz-Aubert pour ses conseils et M. Jean-Yves Mandin pour l'aide importante qu'il leur a apportée dans le domaine de la spectroscopie.

\section{Bibliographie}

[1] Hottel, H. C., SARofim, A. F., Radiative Transfer (Mc Graw Hill, New York) 1967.

[2] Edwards, D. K., Absorption by infrared bands of carbon dioxide gas at elevated pressures and temperatures. J. Opt. Soc. Am. 50 (1960) 617-626.

[3] Edwards, D. K., Molecular gas band radiation. Adv. Heat Transfer 12 (1976) 115-193.

[4] BEN ARYeH, Y., Spectral emissivity calculations by the statistical model applied to the $4,3 \mu \mathrm{m}$ bands of $\mathrm{CO}_{2}$ at high temperatures. Appl. Opt. 6 (1967) 1049-1055.

[5] Bernstein, L. S., Band model parameters for the parallel bands of linear triatomic molecules. I. - Theory. $J$. Quant. Spectrosc. Radiat. Transfer 23 (1980) 157-167.
[6] Bernstein, L. S., Robertson, D. C., Concint, J. A., Band model parameters for the $4,3 \mu \mathrm{m} \mathrm{CO}$ band from 200 to 3000 K. J. Quant. Spectrosc. Radiat. Transfer 23 (1980) 169-185.

[7] Garing, J. S., Mac Latchey, R. A., Atmospheric absorption line compilation. Appl. Opt. 12 (1973) 2545-2545.

[8] Rothman, L. S., Benedict, W. S., Infrared energy and intensities of carbon dioxide. Appl. Opt. 17 (1978) 2605-2611.

[9] BarchewITz, P., Spectroscopie atomique et moléculaire, tomes I et II (Masson, Paris) 1970.

[10] Huetz-AuberT, M., Laterrasse, J., Relaxation thermique de vibration d'un gaz, Application à $\mathrm{CO}_{2}$. J. Physique 35 (1974) 547-555. 
[11] Treanor, C. E., Rich, J. W., ReHm, R. G., Vibrational relaxation of anharmonic oscillators with exchange-dominated collisions. J. Chem. Phys. 48 (1968) 1798-1807.

[12] Burch, D. E., Gryvnak, D. A., Paty, R. R., Bartky, C. E., Absorption of infrared radiant energy by $\mathrm{CO}_{2}$ and $\mathrm{H}_{2} \mathrm{O}$. J. Opt. Soc. Am. 59 (1969) 267-280.

[13] Planet, W. G., Tettemer, G. L., Temperature dependent intensities and widths of $\mathrm{N}_{2}$-broadeed $\mathrm{CO}_{2}$ lines at $15 \mu \mathrm{m}$ from tunable laser measurements. J. Quant. Spectrosc. Radiat. Transfer 22 (1979) 345-354.

[14] Varanasi, P., Measurement of line widths of CO of planetary interest at low temperatures. J. Quant. Spectrosc. Radiat. Transfer 15 (1975) 191-196.

[15] Bouanich, J. P., Broadbeck, C., Mesure des largeurs et des déplacements des raies de la bande $0 \rightarrow 2$ de $\mathrm{CO}$ autoperturbé et perturbé par $\mathrm{N}_{2}, \mathrm{O}_{2}, \mathrm{H}_{2}, \mathrm{HCl}, \mathrm{NO}$ et $\mathrm{CO}_{2}$. J. Quant. Spectrosc. Radiat. Transfer 13 (1973) 1-7.

[16] Sell, J. E., Temperature dependence of the absorption coefficient and half with of the $\mathrm{P}(6)$ line of CO. J. Quant. Spectrosc. Radiat. Transfer 23 (1980) 595-598.

[17] Tettemer, G. L., Planet, W. G., Intensities and pressure broadened widths of $\mathrm{CO}_{2} \mathrm{R}$ branch lines at $15 \mu \mathrm{m} . J$ Quant. Spectrosc. Radiat. Transfer 24 (1980) 343-346.

[18] Galatry, L., Simultaneous effect of Doppler and Foreign Gas Broadening on spectral lines. Phys. Rev. 122 (1961) 1218-1223.

[19] Rodgers, C. D., Collisional narrowing; its effect on the equivalent widths of the spectral lines. Appl. Opt. 15 (1976) 714-716.
[20] Herbert, F., Spectrum line profiles. A generalized voigt function including collisional narrowing. J. Quant. Spectrosc. Radiat. Transfer 14 (1974) 943-951.

[21] Winters, B. H., Silverman, S., Benedict, W. S., Line shape in the wing beyond the band head of the $4,3 \mu$ band of $\mathrm{CO}_{2}$. J. Quant. Spectrosc. Radiat. Transfer 4 (1964) 527-537.

[22] Oppenheim, G. P., Ben Ar yeh, Y., Statistical model applied to the region of the $v_{3}$ fundamental of $\mathrm{CO}_{2}$ at $1200 \mathrm{~K}$. J. Opt. Soc. Am. 53 (1963) 344-350.

[23] Ferriso, C. C., Ludwig, C. B., Acton, L. A., J. Opt. Soc. Am. 56 (1966) 171-173.

[24] SuARez, C. B., Valero, F. P. J., Intensities self broadening and broadening by $\mathrm{Ar}$ and $\mathrm{N}_{2}$ for the $301_{\mathrm{III}} \leftarrow 000$ band of $\mathrm{CO}_{2}$ measured at different temperatures. J. Mol. Spectrosc. 71 (1978) 46-63.

[25] BURCH, D. E., GryvNAK, D. A., Infrared emitted by hot gases and its transmission through synthetic atmosphere. Sci. Rept $\mathrm{n}^{\circ}$ U-1929. Ford Motor Company, Aeronutronic Division, California (1962)

[26] Paramo, F., Thèse de Docteur Ingénieur en thermique E.C.P. Paris, Contribution à l'étude des caractéristiques radiatives de gaz de combustion, déc. 1980.

[27] Bevans, J. T., Dunkde, R. V., Edwards, D. K., Gier, T., LeVenson, L. L., Oppenheim, A. K., Apparatus for the determination of the band absorption of gases at elevated pressures and temperatures. J. Opt. Soc. Am. 50 (1960) $130-136$.

[28] Herzberg, G., Molecular spectra and molecular structure. II. Polyatomic molecules (D. Van Nostrand Company Inc.) 1954. 